\title{
A Highly Enantio- and Diastereoselective Catalytic Intramolecular Stetter Reaction
}

\author{
Javier Read de Alaniz and Tomislav Rovis* \\ Department of Chemistry, Colorado State University \\ Fort Collins, Colorado 80523
}

\section{Supporting Information}

General Methods. All reactions were carried out under atmosphere of argon in flame-dried glassware with magnetic stirring. Column chromatography was performed on EM Science silica gel 60 (230-400 mesh). Thin layer chromatography was performed on EM Science $0.25 \mathrm{~mm}$ silica gel 60-F plates. Visualization was accomplished with $\mathrm{UV}$ light, $\mathrm{KMnO}_{4}$, and aqueous ceric ammonium molybdate dips followed by heating.

Infrared spectra were obtained on a Nicolet Avatar 320 FT-IR spectrometer. ${ }^{1} \mathrm{H}$ NMR and spectra were recorded on a Varian spectrometer at 75 or $100 \mathrm{MHz}$ at ambient temperature. Data are reported as follows: chemical shift in parts per million $(\delta \mathrm{ppm})$ from an internal [standard tetramethylsilane (TMS) or deuterated chloroform $\left(\mathrm{CDCl}_{3}\right)$ ], multiplicity $(\mathrm{s}=$ singlet, $\mathrm{d}$ $=$ doublet, $\mathrm{t}=$ triplet, $\mathrm{q}=$ quartet, and $\mathrm{m}=$ multiplet $)$, integration, and coupling constant $(\mathrm{Hz})$. ${ }^{13} \mathrm{C}$ NMR were recorded on a Varian 300 or $400 \mathrm{MHz}$ spectrometer at ambient temperature. Chemical shifts are reported in ppm from $\left(\mathrm{CDCl}_{3}\right)$ taken as $77.0 \mathrm{ppm}$. Mass spectra were obtained on Fisons VG Autospec. Analytical high performance liquid chromatography (HPLC) was performed on a Dynamax model SD-200 HPLC equipped with a Dynamax model UV-1 variable wavelength UV detector using a Chiracel OD-H, OB-H, OJ, AS $(0.46 \mathrm{~cm}$ X $25 \mathrm{~cm})$ chiral column. Gas Chromatography was performed on a Varian Cp 3800 gas chromatograph equipped with a flame ionization dector using a Chiraldex B-DM and a CP Wax 52CB column. Optical rotations were measured on an Autopol III automatic polarimeter in a $1 \mathrm{dm}$ cell.

Toluene was degassed with argon and passed through one column of neutral alumina and one column of Q5 reactant. KHMDS was purchased from Aldrich Chemical Co. as a $0.5 \mathrm{M}$ solution in toluene and used without purification.

Racemic material was prepared by the method of Ciganek ${ }^{1}$ with the following modifications; $\mathrm{CH}_{2} \mathrm{Cl}_{2}$ was used as the solvent. Preparation of chiral triazolium salts 7, 8 and 9 was recently submitted and accepted. ${ }^{2}$ Relative configuration of $\mathbf{1 1}$ and $\mathbf{2 1}$ has been assigned based on single crystal X-ray analysis. The others were assigned by analogy. The absolute stereochemistry was assigned by analogy to our previous enantioselective Stetter Reaction (ref. 10a in text) lacking the $\alpha$ substituent. Preparation of $\mathbf{3 3}$ and $\mathbf{3 5}$ were prepared from the corresponding aldehyde and HWE reagent and separated on column chromatography using 6:1

\footnotetext{
${ }^{1}$ Ciganek, E. Synthesis 1995, 1311.

${ }^{2}$ Kerr, M. S.; Read de Alaniz, J.; Rovis, T. J. Org. Chem. Manuscript in preparation.
} 
hexane:ethyl acetate. The geometrical isomers were assigned according to the downfield shift of the $\beta$-hydrogen in the ${ }^{1} \mathrm{H}$ NMR according to literature precedent. ${ }^{3}$

General procedure for the asymmetric intramolecular Stetter reaction: A flame dried round bottom flask was charged with triazolium salt $(0.2 \mathrm{eq})$ and $2 \mathrm{~mL}$ of toluene. To this solution was added KHMDS ( $0.5 \mathrm{M}$ in toluene) ( $0.2 \mathrm{eq})$ via syringe and the solution was stirred at ambient temperature for 5 minutes. Toluene and HMDS was removed in vacuo by being placed under high vacuum for 1 hour. ${ }^{4}$ Toluene $(3 \mathrm{~mL})$ was added followed by a solution of the substrate $(1 \mathrm{eq}$ $0.12 \mathrm{mmol}$ ) in $2 \mathrm{~mL}$ of toluene and the resulting solution was allowed to stir at ambient temperature for 24 hours. The reaction was quenched with $15 \% \mathrm{AcOH} /$ toluene $(2 \mathrm{~mL})$ and the resulting solution was purified by flash column chromatography and eluted with a suitable solution of hexane and ethyl acetate (typically 6:1). Evaporation of solvent afforded analytically pure product.

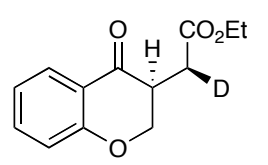

(2S, 3'R)-(4-Oxo-chroman-3-yl-deuterio)-acetic acid ethyl ester (6). According to the general procedure, $13.0 \mathrm{mg}(0.031 \mathrm{mmol})$ of 7 and $61.0 \mu \mathrm{L}$ $(0.031 \mathrm{mmol})$ of KHMDS and $35.0 \mathrm{mg}(0.148 \mathrm{mmol})$ of 5 were reacted for 24 hours. Work-up afforded $31.5 \mathrm{mg}(90 \%)$ of the desired product as colorless oil in $90 \%$ ee and 9:1 dr. $\mathrm{R}_{\mathrm{f}}(1: 1$ hexane to ethyl acetate $)=0.7 ;[\alpha]_{\mathrm{D}}{ }^{23}=+5.27^{\circ}\left(\mathrm{CHCl}_{3}\right) ; \mathrm{HPLC}$ analysis - Chiracel AD column 97:3 hexanes to isopropanol $0.5 \mathrm{~mL} / \mathrm{min}$. Minor enantiomer: 22.95 minutes. Major enantiomer: 32.22 minutes; ${ }^{1} \mathrm{H}$ NMR $\left(400 \mathrm{MHz}, \mathrm{CDCl}_{3}\right) \delta 7.86(1 \mathrm{H}, \mathrm{d}$, $J=7.9 \mathrm{~Hz}), 7.45(1 \mathrm{H}, \mathrm{dd}, J=8.6,8.6 \mathrm{~Hz}), 6.99(1 \mathrm{H}, \mathrm{dd}, J=7.5,7.5 \mathrm{~Hz}), 6.94(1 \mathrm{H}, \mathrm{d}, J=8.3 \mathrm{~Hz})$, $4.57(1 \mathrm{H}, \mathrm{dd}, J=5.3,11.1 \mathrm{~Hz}), 4.27(1 \mathrm{H}, \mathrm{dd}, J=11.6,11.6 \mathrm{~Hz}), 4.16(2 \mathrm{H}, \mathrm{q}, J=7.0 \mathrm{~Hz}), 3.3(1 \mathrm{H}$, m), $2.90(0.2 \mathrm{H}, \mathrm{dd}, J=4.8,16.9 \mathrm{~Hz}), 2.38(1 \mathrm{H}, \mathrm{m}), 1.25(3 \mathrm{H}, \mathrm{t}, J=7.0 \mathrm{~Hz}) ;{ }^{13} \mathrm{C}$ NMR $(100 \mathrm{MHz}$, $\left.\mathrm{CDCl}_{3}\right) \delta 192.8,171.6,161.9,136.2,127.6,121.7,120.7,116.0,70.4,61.2,42.6,30.2(\mathrm{t}, J=20.1$ $\mathrm{Hz}), 14.4$; IR $\left(\mathrm{NaCl}, \mathrm{CH}_{2} \mathrm{Cl}_{2}\right) 1738,1694,1600 \mathrm{~cm}^{-1}$; HRMS (FAB+) calcd for $\mathrm{C}_{13} \mathrm{H}_{13} \mathrm{DO}_{4}$ 235.0954, Found 236.1034.

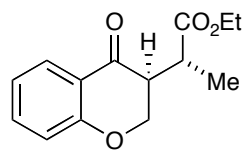

(2R, 3'S)-(4-Oxo-chroman-3-yl)-propionic acid ethyl ester (11). According to the general procedure, $13.0 \mathrm{mg}(0.031 \mathrm{mmol})$ of $\mathbf{8}$ and $61.0 \mu \mathrm{L}(0.031 \mathrm{mmol})$ of KHMDS and $38.0 \mathrm{mg}(0.153 \mathrm{mmol})$ of $\mathbf{1 0}$ were reacted for 24 hours. Work-up afforded $35.7 \mathrm{mg}(94 \%)$ of the desired product as colorless oil in $95 \%$ ee and $30: 1 \mathrm{dr} . \mathrm{R}_{\mathrm{f}}(1: 1$ hexane to ethyl acetate $)=0.7 ;[\alpha]_{\mathrm{D}}^{23}=+7.85^{\circ}\left(\mathrm{CHCl}_{3}\right)$; HPLC analysis - Chiracel OB-H column 97:3 hexanes to isopropanol $0.3 \mathrm{~mL} / \mathrm{min}$. Minor enantiomer: 98.1 minutes. Major enantiomer: 54.9 minutes; GC analysis - $\mathrm{CP}$ Wax $52 \mathrm{CB}$ column $130{ }^{\circ} \mathrm{C}$ at $3 \mathrm{ml} / \mathrm{min}$. Minor diastereomer: 16.7 minutes, Major diastereomer: 19.0 minutes; ${ }^{1} \mathrm{H}$ NMR $\left(400 \mathrm{MHz}, \mathrm{CDCl}_{3}\right) \delta 7.86(1 \mathrm{H}, \mathrm{d}$, $J=7.9 \mathrm{~Hz}), 7.45(1 \mathrm{H}, \mathrm{m}), 6.99(1 \mathrm{H}, \mathrm{m}), 6.94(1 \mathrm{H}, \mathrm{d}, J=8.3 \mathrm{~Hz}), 4.59(1 \mathrm{H}, \mathrm{dd}, J=5.3,11.3 \mathrm{~Hz})$, $4.34(1 \mathrm{H}, \mathrm{dd}, J=11.7,11.7 \mathrm{~Hz}), 4.16(2 \mathrm{H}, \mathrm{q}, J=7.0 \mathrm{~Hz}), 3.26(1 \mathrm{H}, \mathrm{ddd}, J=5.3,5.3,12.2 \mathrm{~Hz})$, $3.10(1 \mathrm{H}, \mathrm{dq}, J=6.0,7.1 \mathrm{~Hz}), 1.25(3 \mathrm{H}, \mathrm{t}, J=7.1 \mathrm{~Hz}), 1.2(3 \mathrm{H}, \mathrm{d}, J=7.2 \mathrm{~Hz}) ;{ }^{13} \mathrm{C}$ NMR $(100$

\footnotetext{
${ }^{3}$ (a) Dieter, R. K.; Silks, L, A.; Fishpaugh, J. R.; Kastner, M. E. J. Am. Chem. Soc. 1985, 107, 4679-4692. (b) Horiuchi, S.; Takikawa, H.; Mori, K. Eur. J. Org. Chem. 1998, 2851-2854. (c) Ballini, R.; Bosica, G.; Mase, A.; Petrini, M. Eur. J. Org. Chem. 2000, 2927-2931, and references therein.

${ }^{4} \mathrm{~A}$ control experiment was run in the absence of $\mathrm{KBF}_{4}$ salt and the enantio and diastereoselectivity of the reaction was not affected. The $\mathrm{KBF}_{4}$ salt was removed by passing a solution of toluene containing the carbene and $\mathrm{KBF}_{4}$ salt, which was prepared according to general procedure, through a Gelman $0.45 \mu \mathrm{m}$ filter.
} 
$\left.\mathrm{MHz}, \mathrm{CDCl}_{3}\right) \delta 192.6,174.9,161.8,136.1,127.6,121.7,122.2,117.9,68.7,61.1,47.8,36.6$, 14.4, 13.7; IR ( $\left.\mathrm{NaCl}, \mathrm{CH}_{2} \mathrm{Cl}_{2}\right)$ 1723, 1701, $1600 \mathrm{~cm}^{-1}$; HRMS (FAB+) calcd for $\mathrm{C}_{14} \mathrm{H}_{16} \mathrm{O}_{4}$ 248.1049, Found 249.1119.

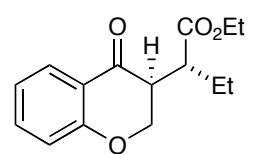

(2R, 3'S)-(4-Oxo-chroman-3-yl)-butyric acid ethyl ester (13). According to the general procedure, $10.0 \mathrm{mg}(0.023 \mathrm{mmol})$ of 8 and $46.0 \mu \mathrm{L}(0.023 \mathrm{mmol})$ of KHMDS and $30.0 \mathrm{mg}(0.116 \mathrm{mmol})$ of $\mathbf{1 2}$ were reacted for 24 hours. Work-up afforded $28.2 \mathrm{mg}(94 \%)$ of the desired product as colorless oil in $92 \%$ ee and 35:1 dr. $\mathrm{R}_{\mathrm{f}}(1: 1$ hexane to ethyl acetate $)=0.7 ;[\alpha]_{\mathrm{D}}^{23}=+8.0^{\circ}\left(\mathrm{CHCl}_{3}\right)$; HPLC analysis - Chiracel AS column 95:5 hexanes to isopropanol $0.5 \mathrm{~mL} / \mathrm{min}$. Minor enantiomer: 12.7 minutes. Major enantiomer: 13.6 minutes; $\mathrm{GC}$ analysis - CP Wax $52 \mathrm{CB}$ column $130^{\circ} \mathrm{C}$ at $3 \mathrm{ml} / \mathrm{min}$. Major diastereomer: 26.5 minutes, Minor diastereomer: 23.2 minutes; ${ }^{1} \mathrm{H}$ NMR $\left(400 \mathrm{MHz}, \mathrm{CDCl}_{3}\right) \delta 7.85(1 \mathrm{H}, \mathrm{d}, J=7.9$ $\mathrm{Hz}), 7.44(1 \mathrm{H}, \mathrm{m}), 6.99(1 \mathrm{H}, \mathrm{m}), 6.92(1 \mathrm{H}, \mathrm{d}, J=8.3 \mathrm{~Hz}), 4.49(1 \mathrm{H}, \mathrm{dd}, J=5.1,11.5 \mathrm{~Hz}), 4.32$ $(1 \mathrm{H}, \mathrm{dd}, J=11.5,11.5 \mathrm{~Hz}), 4.16(2 \mathrm{H}, \mathrm{q}, J=7.0 \mathrm{~Hz}), 3.17(1 \mathrm{H}, \mathrm{ddd}, J=5.1,6.4,11.7 \mathrm{~Hz}), 2.85$ $(1 \mathrm{H}, \mathrm{ddd}, J=4.7,6.2,10.3 \mathrm{~Hz}), 1.71-1.51(2 \mathrm{H}, \mathrm{m}), 1.24(3 \mathrm{H}, \mathrm{t}, J=7.1 \mathrm{~Hz}), 0.93(3 \mathrm{H}, \mathrm{t}, J=7.4$ $\mathrm{Hz}) ;{ }^{13} \mathrm{C}$ NMR $\left(100 \mathrm{MHz}, \mathrm{CDCl}_{3}\right) \delta 192.6,174.4,161.7,136.1,127.6,121.7,121.0,117.9,68.9$, 60.9, 47.4, 43.9, 22.0, 14.4, 12.2; IR( $\left.\mathrm{NaCl}, \mathrm{CH}_{2} \mathrm{Cl}_{2}\right)$; HRMS (FAB+) calcd for $\mathrm{C}_{15} \mathrm{H}_{18} \mathrm{O}_{4}$ 262.1205, Found 263.1293.

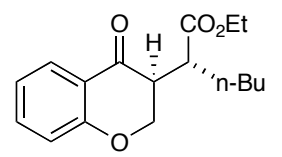

(2R, 3'S)-(4-Oxo-chromoan-3yl)-hexanoic acid ethyl ester (15). According to the general procedure, $5.0 \mathrm{mg}(0.012 \mathrm{mmol})$ of 8 and $23.0 \mu \mathrm{L}(0.012 \mathrm{mmol})$ of KHMDS and $33.0 \mathrm{mg}(0.116 \mathrm{mmol})$ of 15 were reacted for 12 hours. A second portion of a solution of $\mathbf{8}$ prepared according to the general procedure was added and the reaction was reacted for 12 additional hours. Work-up afforded $17.5 \mathrm{mg}(53 \%)$ of the desired product as colorless oil in $94 \%$ ee and $12: 1 \mathrm{dr}$. $\mathrm{R}_{\mathrm{f}}(4: 1$ hexane to ethyl acetate $)=0.5 ;[\alpha]_{\mathrm{D}}{ }^{23}=+$ 7.0 $0^{\circ}\left(\mathrm{CHCl}_{3}\right)$; GC analysis- Chiraldex B-DM $150^{\circ} \mathrm{C}$ at $3 \mathrm{~mL} / \mathrm{min}$. Major enantiomer: 158.4 minutes, Minor enantiomer: 155.9 minutes; $\mathrm{GC}$ analysis- $\mathrm{CP}$ Wax $52 \mathrm{CB}$ column $130^{\circ} \mathrm{C}$ at 3ml/min. Major diastereomer: 60.3 minutes, Minor diastereomer: 53.1 minutes; ${ }^{1} \mathrm{H}$ NMR (400 $\mathrm{MHz}), \delta 7.86(1 \mathrm{H}, \mathrm{dd}, J=1.5,7.9 \mathrm{~Hz}), 7.45(1 \mathrm{H}, \mathrm{m}), 6.99(1 \mathrm{H}, \mathrm{m}), 6.94(1 \mathrm{H}, \mathrm{d}, J=8.1 \mathrm{~Hz}), 4.60$ $(1 \mathrm{H}, \mathrm{dd}, J=5.0,11.4 \mathrm{~Hz}), 4.34(1 \mathrm{H}, \mathrm{d}, 11.5 \mathrm{~Hz}), 4.16(2 \mathrm{H}, \mathrm{qq}, J=1.7,7.1 \mathrm{~Hz}), 3.16(1 \mathrm{H}, \mathrm{ddd}, J=$ $5.1,11.5,5.1 \mathrm{~Hz}), 2.95(1 \mathrm{H}, \mathrm{ddd}, J=5.9,10.2,10.2 \mathrm{~Hz}), 1.34-1.28(6 \mathrm{H}, \mathrm{m}), 1.25(3 \mathrm{H}, \mathrm{t}, J=7.2$ $\mathrm{Hz}), 0.87(3 \mathrm{H}, \mathrm{m}) ;{ }^{13} \mathrm{C} \mathrm{NMR}\left(100 \mathrm{MHz}, \mathrm{CDCl}_{3}\right) \delta 192.5,174.6,161.8,136.1,127.6,121.7$, 121.0, 117.9, 68.9, 60.9, 47.7, 42.4, 29.9, 28.5, 22.7, 14.4, 14.1; IR ( $\left.\mathrm{NaCl}, \mathrm{CH}_{2} \mathrm{Cl}_{2}\right)$ 1730, 1688, 1600, 1457; HRMS (FAB+) calcd for $\mathrm{C}_{17} \mathrm{H}_{22} \mathrm{O}_{4}$ 290.1518. Found 291.1590 .

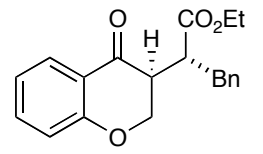

(2R, 3'S)-(4-Oxo-chromoan-3yl)-phenyl-propionic acid ethyl ester (17). According to the general procedure, $10.0 \mathrm{mg}(0.023 \mathrm{mmol})$ of 8 and $46.0 \mu \mathrm{L}$ $(0.023 \mathrm{mmol})$ of KHMDS and $37.0 \mathrm{mg}(0.116 \mathrm{mmol})$ of 16 were reacted for 24 hours. Work-up afforded $30.0 \mathrm{mg}(80 \%)$ of the desired product as colorless oil in $84 \%$ ee and $20: 1 \mathrm{dr} . \mathrm{R}_{\mathrm{f}}(1: 1$ hexane to ethyl acetate $)=0.6 ;[\alpha]_{\mathrm{D}}^{23}=+39.5^{\circ}\left(\mathrm{CHCl}_{3}\right) ; \mathrm{HPLC}$ analysis Chiracel OJ column 90:10 hexanes to isopropanol $1.0 \mathrm{~mL} / \mathrm{min}$. Minor enantiomer: 13.83 minutes, Major enantiomer: 17.49 minutes; ${ }^{1} \mathrm{H}\left(400 \mathrm{MHz}, \mathrm{CDCl}_{3}\right) \delta 7.89(1 \mathrm{H}, \mathrm{dd}, J=1.5,7.9$ $\mathrm{Hz}), 7.45(1 \mathrm{H}, \mathrm{m}), 7.27-7.18(5 \mathrm{H}, \mathrm{m}), 7.00(1 \mathrm{H}, \mathrm{dd}, J=7.7,7.7 \mathrm{~Hz}), 6.94,(1 \mathrm{H}, \mathrm{d}, J=8.4 \mathrm{~Hz})$, $4.58(1 \mathrm{H}, \mathrm{dd}, J=5.1,11.5 \mathrm{~Hz}), 4.49(1 \mathrm{H}, \mathrm{dd}, J=11.5,15.4 \mathrm{~Hz}), 4.02(2 \mathrm{H}, \mathrm{m}), 3.19(1 \mathrm{H}, \mathrm{m}), 3.17$ $(1 \mathrm{H}, \mathrm{m}), 3.00(1 \mathrm{H}, \mathrm{m}), 2.90(1 \mathrm{H}, \mathrm{m}), 1.07(3 \mathrm{H}, \mathrm{t}, J=7.0 \mathrm{~Hz}) ;{ }^{13} \mathrm{C} \mathrm{NMR}\left(100 \mathrm{MHz}, \mathrm{CDCl}_{3}\right) \delta$ 192.4, 174.8, 173.5, 161.8, 139.0, 136.2, 129.3, 129.2, 128.7, 127.6, 126.8, 122.8, 121.3, 117.9, 
69.3, 61.0, 47.3, 45.1, 35.0, 14.2; IR $\left(\mathrm{NaCl}, \mathrm{CH}_{2} \mathrm{Cl}_{2}\right)$ 1730, 1687, 1607; HRMS (FAB+) calcd for $\mathrm{C}_{20} \mathrm{H}_{20} \mathrm{O}_{4}$ 324.1362. Found 325.1439.

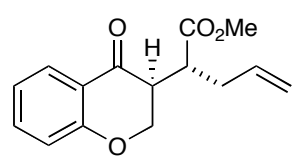

(2R, 3'S)-(4-Oxo-chroman-3-yl)-pent-4-enoic acid methyl ester (19). According to the general procedure, $13.0 \mathrm{mg}(0.030 \mathrm{mmol})$ of $\mathbf{8}$ and $61.0 \mu \mathrm{L}$ $(0.030 \mathrm{mmol})$ of KHMDS and $40.0 \mathrm{mg}(0.154 \mathrm{mmol})$ of 18 were reacted for 24 hours. Work-up afforded $36.0 \mathrm{mg}(90 \%)$ of the desired product as colorless oil in $83 \%$ ee and13:1 dr. $\mathrm{R}_{\mathrm{f}}(1: 1$ hexane to ethyl acetate $)=0.6 ;[\alpha]_{\mathrm{D}}^{23}=+40.4^{\circ}\left(\mathrm{CHCl}_{3}\right) ; \mathrm{HPLC}$ anaylsis - Chiracel OD-H 97:3 hexanes to isopropanol $0.3 \mathrm{~mL} / \mathrm{min}$. Minor enantiomer: 17.4 minutes, Major enantiomer: 22.2 minutes; GC analysis - $\mathrm{CP}$ Wax $52 \mathrm{CB}$ column $130^{\circ} \mathrm{C}$ at 3ml/min. Major diastereomer: 27.3 minutes, Minor diastereomer: 24.3 minutes; ${ }^{1} \mathrm{H}(400 \mathrm{MHz}$, $\left.\mathrm{CDCl}_{3}\right) \delta 7.84(1 \mathrm{H}, \mathrm{d}, J=7.9 \mathrm{~Hz}), 7.44(1 \mathrm{H}, \mathrm{m}), 6.99(1 \mathrm{H}, \mathrm{m}), 6.93(1 \mathrm{H}, \mathrm{d}, J=8.3 \mathrm{~Hz}), 5.73(1 \mathrm{H}$, dddd, $J=7.0,10.2,14.0,17.2 \mathrm{~Hz}), 5.06(2 \mathrm{H}, \mathrm{dd}, J=17.0,20.4), 4.59(1 \mathrm{H}, \mathrm{dd}, J=5.1,11.3) 4.37$ (1H, dd, $J=11.6,11.6), 3.68(3 \mathrm{H}, \mathrm{s}), 3.21(1 \mathrm{H}, \mathrm{ddd}, J=5.7,11.7,5.7), 3.0(1 \mathrm{H}, \mathrm{m}), 2.38(1 \mathrm{H}, \mathrm{m})$; ${ }^{13} \mathrm{C}$ NMR $\left(100 \mathrm{MHz}, \mathrm{CDCl}_{3}\right) \delta 192.4,174.1,161.7,136.1,134.8,127.6,121.7,121.0,117.9$, 68.9, 52.1, 46.9, 42.2, 33.3; IR ( $\left.\mathrm{NaCl}, \mathrm{CH}_{2} \mathrm{Cl}_{2}\right)$ 1731, 1690, 1603, 1480, 1209; HRMS (FAB+) calcd for $\mathrm{C}_{15} \mathrm{H}_{16} \mathrm{O}_{4}$ 260.1049. Found 261.1138.

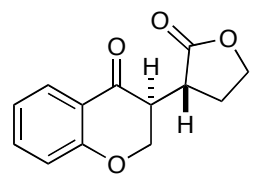

(3R, 3'R)-(2-Oxo-tetrahydro-furan-3yl)-chroman-4-one (21). According to the general procedure, $10.0 \mathrm{mg}(0.023 \mathrm{mmol})$ of $\mathbf{8}$ and $46.0 \mu \mathrm{L}(0.023 \mathrm{mmol})$ of KHMDS and $27.0 \mathrm{mg}(0.116 \mathrm{mmol})$ of $\mathbf{2 0}$ were reacted for 24 hours. Work-up afforded $25.6 \mathrm{mg}(95 \%)$ of the desired product as a white solid in $94 \%$ ee and $10: 1$ dr. $\mathrm{R}_{\mathrm{f}}(1: 1$ hexane to ethyl acetate $)=0.4 ;[\alpha]_{\mathrm{D}}{ }^{23}=+17.0^{\circ}\left(\mathrm{CHCl}_{3}\right)$; HPLC analysis Chiracel OD-H column 90:10 hexanes to isopropanol $1.0 \mathrm{~mL} /$ minute Major enantiomer: 27.5 minutes, Minor enantiomer: 31.6 minutes; GC analysis - $\mathrm{CP}$ Wax $52 \mathrm{CB}$ column $130{ }^{\circ} \mathrm{C}$ at 3 $\mathrm{ml} / \mathrm{min}$. Minor diastereomer: 46.6 minutes, Major diastereomer: 49.9 minutes; ${ }^{1} \mathrm{H}(400 \mathrm{MHz}$, $\left.\mathrm{CDCl}_{3}\right) \delta 7.86(1 \mathrm{H}, \mathrm{dd}, J=1.7,7.9 \mathrm{~Hz}), 7.47(1 \mathrm{H}, \mathrm{m}), 7.01(1 \mathrm{H}, \mathrm{m}), 6.95(1 \mathrm{H}, \mathrm{d}, J=8.3 \mathrm{~Hz})$, 4.57-4.44 (3H, m), $4.25(1 \mathrm{H}, \mathrm{ddd}, J=6.6,9.8,16.6 \mathrm{~Hz}), 3.42(1 \mathrm{H}, \mathrm{ddd}, J=2.6,6.4,11.5 \mathrm{~Hz})$, $3.19(1 \mathrm{H}, \mathrm{ddd}, J=3.1,9.1,11.9 \mathrm{~Hz}), 2.41(1 \mathrm{H}, \mathrm{m}), 2.25(1 \mathrm{H}, \mathrm{m}) ;{ }^{13} \mathrm{C}$ NMR $\left(100 \mathrm{MHz}, \mathrm{CDCl}_{3}\right) \delta$ 192.4, 177.7, 161.9, 136.5, 127.5, 121.8, 121.0, 118.1, 68.6, 67.1, 45.3, 37.6, 25.9; IR (NaCl, $\mathrm{CH}_{2} \mathrm{Cl}_{2}$ ) 1760, 1680, 1600; HRMS (FAB+) calcd for $\mathrm{C}_{13} \mathrm{H}_{12} \mathrm{O}_{4}$ 232.0736. Found 233.0819.

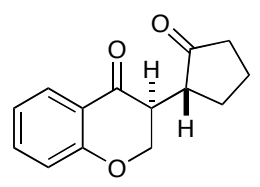

(3R, 3'S)-(2-Oxo-cyclopentyl)-chroman-4-one (23). According to the general procedure, $10.0 \mathrm{mg}(0.023 \mathrm{mmol})$ of $\mathbf{8}$ and $46.0 \mu \mathrm{L}(0.023 \mathrm{mmol})$ of KHMDS and $27.0 \mathrm{mg}(0.115 \mathrm{mmol})$ of 22 were reacted for 24 hours. Work-up afforded $21.6 \mathrm{mg}(80 \%)$ of the desired product as a white solid in $95 \%$ ee and $18: 1 \mathrm{dr} . \mathrm{R}_{\mathrm{f}}$ $(1: 1$ hexane to ethyl acetate $)=0.4 ;[\alpha]_{\mathrm{D}}^{23}=+117^{\circ}\left(\mathrm{CHCl}_{3}\right) ; \mathrm{HPLC}$ analysis - Chiracel AS column 95:5 hexanes to isopropanol $1 \mathrm{~mL} / \mathrm{min}$. Minor enantiomer: 13.5 minutes, Major enantiomer: 34.0 minutes; GC analysis - CP Wax 52CB column $130{ }^{\circ} \mathrm{C}$ at $3 \mathrm{ml} / \mathrm{min}$. Minor diastereomer: 33.5 minutes, Major diastereomer: 32.6 minutes; ${ }^{1} \mathrm{H} \mathrm{NMR}\left(400 \mathrm{MHz}, \mathrm{CDCl}_{3}\right) \delta$ $7.86(1 \mathrm{H}, \mathrm{dd}, J=1.5,7.7 \mathrm{~Hz}), 7.46(1 \mathrm{H}, \mathrm{m}), 7.00(1 \mathrm{H}, \mathrm{m}), 6.95(1 \mathrm{H}, \mathrm{d}, J=8.4 \mathrm{~Hz}), 4.52(1 \mathrm{H}$, dd, $J=10.9,12.5 \mathrm{~Hz}), 4.39(1 \mathrm{H}, \mathrm{dd}, J=5.5,10.9 \mathrm{~Hz}), 3.36(1 \mathrm{H}, \mathrm{ddd}, J=2.6,5.512 .5 \mathrm{~Hz}), 2.65(1 \mathrm{H}$, m), 2.43-2.07 (4H, m), 1.94-1.78 (2H, m); $\left.{ }^{13} \mathrm{C} \mathrm{NMR} \mathrm{(100} \mathrm{MHz,} \mathrm{CDCl}_{3}\right) \delta 219.3,193.4,161.8$, 136.2, 127.5, 121.5, 121.2, 118.0, 69.2, 47.3, 45.6, 38.2, 27.1, 21.5; IR $\left(\mathrm{NaCl}, \mathrm{CH}_{2} \mathrm{Cl}_{2}\right) 1730$, 1683, 1604; HRMS (FAB+) calcd for $\mathrm{C}_{14} \mathrm{H}_{14} \mathrm{O}_{3}$ 230.094. Found 231.1009 


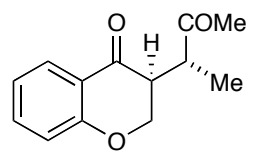

(2R, 3'S)-(1-Methyl-2-oxy-propy)-chroman-4-one (25). According to the general procedure, $10.0 \mathrm{mg}(0.023 \mathrm{mmol})$ of 8 and $46.0 \mu \mathrm{L}(0.023 \mathrm{mmol})$ of KHMDS and $25.0 \mathrm{mg}(0.116 \mathrm{mmol})$ of $\mathbf{2 4}$ were reacted for 24 hours. Work-up afforded $21.0 \mathrm{mg}(85 \%)$ of the desired product as a white solid in $55 \%$ ee and 10:1 dr. $\mathrm{R}_{\mathrm{f}}(1: 1$ hexane to ethyl acetate $)=0.4 ;[\alpha]_{\mathrm{D}}^{23}=-9.5^{\circ}\left(\mathrm{CHCl}_{3}\right)$; GC analysis - Chiraldex B-DM column $150{ }^{\circ} \mathrm{C}$ at $1.5 \mathrm{~mL} / \mathrm{min}$. Minor: 57.0 minutes, 58.0 minutes, Major: 75.0 minutes, 76.0 minutes; ${ }^{1}$ $\mathrm{H}\left(400 \mathrm{MHz}, \mathrm{CDCl}_{3}\right) \delta 7.82(1 \mathrm{H}, \mathrm{dd}, J=1.6,7.9 \mathrm{~Hz}), 7.45(1 \mathrm{H}, \mathrm{m}), 6.98(1 \mathrm{H}, \mathrm{m}), 6.94(1 \mathrm{H}, \mathrm{d}$, $J=8.7 \mathrm{~Hz}), 4.58(1 \mathrm{H}, \mathrm{dd}, J=5.4,11.1 \mathrm{~Hz}), 4.22(1 \mathrm{H}, \mathrm{dd}, J=11.1,13.2 \mathrm{~Hz}), 3.37(1 \mathrm{H}, \mathrm{ddd}, J=$ $5.3,6.9,12.8 \mathrm{~Hz}), 3.07(1 \mathrm{H}, \mathrm{dq}, J=7.2,14.5 \mathrm{~Hz}), 2.31(3 \mathrm{H}, \mathrm{s}), 1.12(3 \mathrm{H}, \mathrm{d}, J=7.3 \mathrm{~Hz}) ;{ }^{13} \mathrm{C}$ NMR $\left(100 \mathrm{MHz}, \mathrm{CDCl}_{3}\right) \delta 210.2,193.5,174.8,161.9,136.2,127.5,121.6,117.9,68.8,47.5$, 43.1, 28.9, 13.3; IR $\left(\mathrm{NaCl}, \mathrm{CH}_{2} \mathrm{Cl}_{2}\right) 1716,1688,1609$; HRMS (FAB+) calcd for $\mathrm{C}_{13} \mathrm{H}_{14} \mathrm{O}_{3}$ 218.0943. Found 219.1027

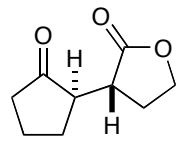

(3R, 2'R)-(2-Oxo-cyclopentyl)-dihydro-furan-2-one (27). ${ }^{5}$ According to the general procedure, $10.0 \mathrm{mg}(0.023 \mathrm{mmol})$ of 8 and $46.0 \mu \mathrm{L}(0.023 \mathrm{mmol})$ of KHMDS and $20.0 \mathrm{mg}(0.116 \mathrm{mmol})$ of $\mathbf{2 6}$ were reacted for 24 hours. Work-up afforded $19.0 \mathrm{mg}(94 \%)$ of the desired product as colorless oil in $99 \%$ ee and 50:1 $\mathrm{dr} . \mathrm{R}_{\mathrm{f}}(1: 1$ hexane to ethyl acetate $)=0.2 ;[\alpha]_{\mathrm{D}}{ }^{23}=-104.4^{\circ}\left(\mathrm{CHCl}_{3}\right)$; GC analysis - Chiraldex B-DM column $150{ }^{\circ} \mathrm{C}$ at $3 \mathrm{~mL} / \mathrm{min}$. Major: 20.3 minutes, 24.23 minutes, Minor: 25.0 minutes, 26.7 minutes; ${ }^{1} \mathrm{H}$ $\left(400 \mathrm{MHz}, \mathrm{CDCl}_{3}\right) \delta 4.36(1 \mathrm{H}, \mathrm{ddd}, J=1.9,8.9,10.8 \mathrm{~Hz}), 4.18(1 \mathrm{H}, \mathrm{ddd}, J=6.6,9.2,10.3 \mathrm{~Hz})$, $3.13(1 \mathrm{H}$, ddd, $J=3.2,9.2,11.9 \mathrm{~Hz}), 2.37(1 \mathrm{H}, \mathrm{m}), 2.26-2.13(2 \mathrm{H}, \mathrm{m}), 2.10-2.02(3 \mathrm{H}, \mathrm{m}), 1.84-$ $1.69(2 \mathrm{H}, \mathrm{m}) ;{ }^{13} \mathrm{C} \mathrm{NMR}\left(100 \mathrm{MHz}, \mathrm{CDCl}_{3}\right) \delta 218.6,178.6,66.9,49.0,38.5,37.9,25.1,24.7$, 20.6; IR ( $\left.\mathrm{NaCl}, \mathrm{CH}_{2} \mathrm{Cl}_{2}\right)$ 1781, 1730; HRMS (FAB+) calcd for $\mathrm{C}_{9} \mathrm{H}_{12} \mathrm{O}_{3}$ 168.0786. Found 169.0865

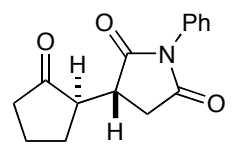

(3R, 2'R)-(2-Oxo-cyclopentyl)-1-phenyl-pyrrolidine-2,5-dione (29). According to the general procedure, $15.0 \mathrm{mg}(0.035 \mathrm{mmol})$ of $\mathbf{8}$ and $68.0 \mu \mathrm{L}(0.035 \mathrm{mmol})$ of KHMDS and $44.0 \mathrm{mg}(0.174 \mathrm{mmol})$ of $\mathbf{2 8}$ were reacted for 24 hours. Work-up afforded $35.0 \mathrm{mg}(80 \%)$ of the desired product as white solid in $88 \%$ ee and $15: 1 \mathrm{dr}$. $\mathrm{R}_{\mathrm{f}}$ (ethyl acetate $)=0.5 ;[\alpha]_{\mathrm{D}}{ }^{23}=-111.64^{\circ}\left(\mathrm{CHCl}_{3}\right) ; \mathrm{GC}$ analysis - Chiraldex B-DM column $170{ }^{\circ} \mathrm{C}$ at 4 $\mathrm{mL} / \mathrm{min}$ for 60 minutes then ramp to $180^{\circ} \mathrm{C}$ over 15 minutes, Major enantiomer: 123.2 minutes, Minor enantiomer: 128.2 minutes. GC analysis - CP Wax $52 \mathrm{CB}$ column $130^{\circ} \mathrm{C}$ at $3 \mathrm{ml} / \mathrm{min}$. Minor diastereomer: 48.5 minutes, Major diastereomer: 38.3 minutes; ${ }^{1} \mathrm{H}\left(400 \mathrm{MHz}, \mathrm{CDCl}_{3}\right) \delta$ 7.46-7.42 (2H, m), 7.36 (1H, m), 7.24-7.22 (2H, m) $3.43(1 \mathrm{H}, \mathrm{ddd}, J=3.2,5.1,9.4 \mathrm{~Hz}), 2.96(1 \mathrm{H}$, $\mathrm{dd}, J=9.6,18.3), 2.82(1 \mathrm{H}, \mathrm{m}), 2.56(1 \mathrm{H}, \mathrm{dd}, J=5.3,23.6 \mathrm{~Hz}), 2.39(1 \mathrm{H}, \mathrm{m}), 2.22-2.04(3 \mathrm{H}, \mathrm{m})$, 1.92-1.80 (2H, m); ${ }^{13} \mathrm{C}$ NMR $\left(100 \mathrm{MHz} \mathrm{CDCl}_{3}\right) \delta$ 218.3, 178.2, 175.3, 131.9, 129.4, 128.9, 126.7, 50.3, 38.9, 37.9, 32.5, 25.5, 20.5; IR $\left(\mathrm{NaCl}_{1} \mathrm{CH}_{2} \mathrm{Cl}_{2}\right)$ 1703, 1507, 1382, 1181; HRMS $(\mathrm{FAB}+)$ calcd for $\mathrm{C}_{15} \mathrm{H}_{15} \mathrm{NO}_{3} 257.1052$. Found 258.1122 .

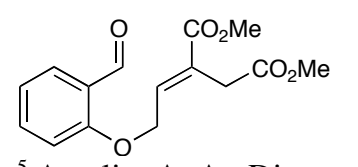

(E)-2-[2-(2-Formyl-phenoxy)-ethylidene]-succinic acid dimethyl ester (33). ${ }^{1} \mathrm{H}\left(400 \mathrm{MHz}, \mathrm{CDCl}_{3}\right) \delta 10.48(1 \mathrm{H}, \mathrm{s}), 7.86(1 \mathrm{H}, \mathrm{dd}, J=1.8,7.7 \mathrm{~Hz})$,

${ }^{5}$ Asselin, A. A.; Dionne, G.; Humber, L. G. U.S. Patent 79 64343, 1980; American Home Products Corp., 1980, 12. 
$7.54(1 \mathrm{H}, \mathrm{m}), 7.17(1 \mathrm{H}, \mathrm{t}, J=5.5 \mathrm{~Hz}), 7.07(1 \mathrm{H}, \mathrm{dd}, J=7.7,7.7 \mathrm{~Hz}), 6.95(1 \mathrm{H}, \mathrm{d}, J=8.4 \mathrm{~Hz}), 4.87$ $(2 \mathrm{H}, \mathrm{d}, J=5.5 \mathrm{~Hz}), 3.79(3 \mathrm{H}, \mathrm{s}), 3.70(3 \mathrm{H}, \mathrm{s}) ; \mathrm{R}_{\mathrm{f}}(1: 1$ hexane:ethyl acetate $)=0.7$.

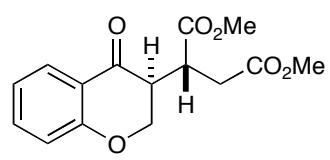

(2R, 3'S)(4-Oxo-chroman-3-yl)-succinic acid dimethyl ester (34). According to the general procedure, $10.0 \mathrm{mg}(0.023 \mathrm{mmol})$ of $\mathbf{8}$ and 46.0 $\mu \mathrm{L}(0.023 \mathrm{mmol})$ of KHMDS and $34.0 \mathrm{mg}(0.115 \mathrm{mmol})$ of $\mathbf{3 3}$ were reacted for 24 hours. Work-up afforded $27.0 \mathrm{mg}(80 \%)$ of the desired product as oil in $92 \%$ ee and $42: 1 \mathrm{dr}$. $\mathrm{R}_{\mathrm{f}}(1: 1$ hexane:ethyl acetate $)=0.8 ;[\alpha]_{\mathrm{D}}{ }^{23}=+48.5^{\circ}\left(\mathrm{CHCl}_{3}\right)$; HPLC analysis - Chiracel AS column 95:5 hexanes to isopropanol $0.5 \mathrm{~mL} / \mathrm{min}$. Major enatiomer: 45.9 minutes, Minor enantiomer: 43.3 minutes; GC analysis - CP Wax 52CB column $130{ }^{\circ} \mathrm{C}$ at $3 \mathrm{ml} / \mathrm{min}$. Minor diastereomer: 71.0 minutes, Major diastereomer: 75.0 minutes; ${ }^{1} \mathrm{H}$ $\left(400 \mathrm{MHz}, \mathrm{CDCl}_{3}\right) \delta 7.84(1 \mathrm{H}, \mathrm{dd}, J=1.7,7.9 \mathrm{~Hz}), 7.45(1 \mathrm{H}, \mathrm{m}), 6.99(1 \mathrm{H}, \mathrm{m}), 6.93(1 \mathrm{H}, \mathrm{d}$, $J=8.3 \mathrm{~Hz}), 4.54-4.43(2 \mathrm{H}, \mathrm{m}), 3.69(3 \mathrm{H}, \mathrm{s}), 3.66(3 \mathrm{H}, \mathrm{s}), 3.47(1 \mathrm{H}, \mathrm{ddd}, J=3.8,5.3,8.7 \mathrm{~Hz}), 3.24$ $(1 \mathrm{H}, \mathrm{ddd}, J=3.6,5.5,11.7 \mathrm{~Hz}), 2.88(1 \mathrm{H}, \mathrm{dd}, J=8.7,16.8 \mathrm{~Hz}), 2.58(1 \mathrm{H}, \mathrm{dd}, J=5.3,16.8 \mathrm{~Hz}) ;{ }^{13} \mathrm{C}$ NMR $\left(100 \mathrm{MHz}, \mathrm{CDCl}_{3}\right) \delta 191.9,173.1,172.0,161.6,136.2,127.3,121.6,120.8,117.8,69.0$, 52.4, 47.0, 38.4, 33.1; IR (NaCl, $\left.\mathrm{CH}_{2} \mathrm{Cl}_{2}\right)$ 1730, 1687, 1600, 1483; HRMS (FAB+) calcd for $\mathrm{C}_{15} \mathrm{H}_{16} \mathrm{O}_{6}$ 292.0947. Found 293.1015.

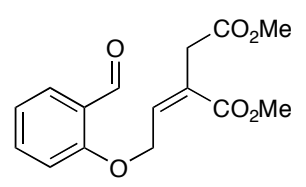

(Z)-2-[2-(2-Formyl-phenoxy)ethylidene]-succinic acid dimethyl ester (35). ${ }^{1} \mathrm{H}\left(400 \mathrm{MHz}, \mathrm{CDCl}_{3}\right) \delta 10.49(1 \mathrm{H}, \mathrm{s}), 7.83(1 \mathrm{H}, \mathrm{dd}, J=1.8,7.7 \mathrm{~Hz})$, $7.52(1 \mathrm{H}, \mathrm{m}), 7.03(1 \mathrm{H}, \mathrm{dd}, J=7.7,7.7 \mathrm{~Hz}), 6.97(1 \mathrm{H}, \mathrm{d}, J=8.4 \mathrm{~Hz}), 6.39$ $(1 \mathrm{H}, \mathrm{t}, 4.5 \mathrm{~Hz}), 5.21(2 \mathrm{H}, \mathrm{d}, J=4.4 \mathrm{~Hz}), 3.78(3 \mathrm{H}, \mathrm{s}), 3.69(3 \mathrm{H}, \mathrm{s}) ; \mathrm{R}_{\mathrm{f}}(1: 1$ hexane:ethyl acetate) $=0.8$.

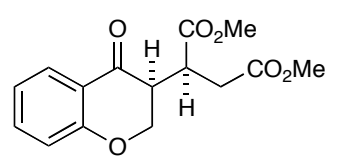

(2S, 3'S)(4-Oxo-chroman-3-yl)-succinic acid dimethyl ester (36). According to the general procedure, $10.0 \mathrm{mg}(0.023 \mathrm{mmol})$ of $\mathbf{8}$ and 46.0 $\mu \mathrm{L}(0.023 \mathrm{mmol})$ of KHMDS and $34.0 \mathrm{mg}(0.115 \mathrm{mmol})$ of $\mathbf{3 5}$ were reacted for 24 hours. Work-up afforded $23.8 \mathrm{mg}(70 \%)$ of the desired product as oil in $38 \%$ ee and 1:6 dr. $\mathrm{R}_{\mathrm{f}}(1: 1$ hexane:ethyl acetate $)=0.8 ;[\alpha]_{\mathrm{D}}{ }^{23}=+9.2^{\circ}\left(\mathrm{CHCl}_{3}\right)$; HPLC analysis - Chiracel AS column 95:5 hexanes to isopropanol $0.5 \mathrm{~mL} / \mathrm{min}$. Major enatiomer: 59.5 minutes, Minor enantiomer: 55.6 minutes; GC analysis - CP Wax 52CB column $130^{\circ} \mathrm{C}$ at $3 \mathrm{ml} / \mathrm{min}$. Minor diastereomer: 75.0 minutes, Major diastereomer: 71.5 minutes; ${ }^{1} \mathrm{H}$ $\left(400 \mathrm{MHz}, \mathrm{CDCl}_{3}\right) \delta 7.85(1 \mathrm{H}, \mathrm{d}, J=7.9 \mathrm{~Hz}), 7.46(1 \mathrm{H}, \mathrm{m}), 7.00(1 \mathrm{H}, \mathrm{m}), 6.94(1 \mathrm{H}, \mathrm{d}, J=9.2 \mathrm{~Hz})$, $4.49(1 \mathrm{H}, \mathrm{dd}, J=5.2,11.2 \mathrm{~Hz}), 4.41(1 \mathrm{H}, \mathrm{dd}, J=11.8,22.9 \mathrm{~Hz}), 3.68(3 \mathrm{H}, \mathrm{s}), 3.65(3 \mathrm{H}, \mathrm{s}), 3.46$ $(1 \mathrm{H}, \mathrm{m}), 3.17(1 \mathrm{H}, \mathrm{ddd}, J=5.3,5.3,10.0 \mathrm{~Hz}), 2.91(1 \mathrm{H}, \mathrm{dd}, J=8.6,17.0 \mathrm{~Hz}), 2.52(1 \mathrm{H}, \mathrm{dd}, J=5.5$, $17.0 \mathrm{~Hz}) ;{ }^{13} \mathrm{C}$ NMR $\left(100 \mathrm{MHz}, \mathrm{CDCl}_{3}\right) \delta 191.8,172.8,172.0,161.4,136.2,127.5,1201.7,117.8$, 68.8, 52.4, 46.9, 38.2, 33.3; IR ( $\left.\mathrm{NaCl}, \mathrm{CH}_{2} \mathrm{Cl}_{2}\right)$ 1735, 1681, 1600; HRMS (FAB+) calcd for $\mathrm{C}_{15} \mathrm{H}_{16} \mathrm{O}_{6}$ 292.0947. Found 293.1015. 


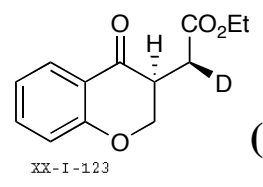

(6)

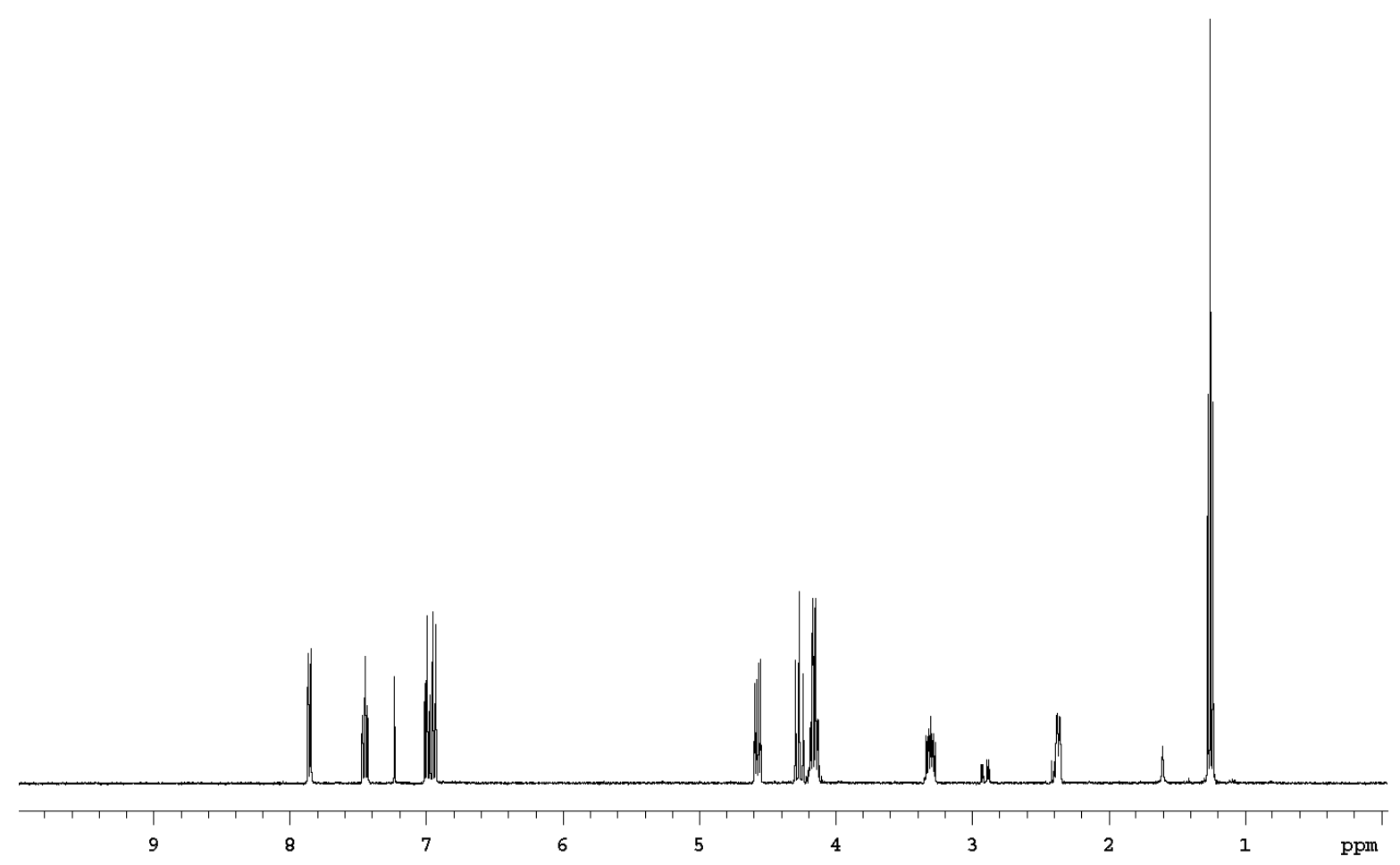

XX-I -123-carbon

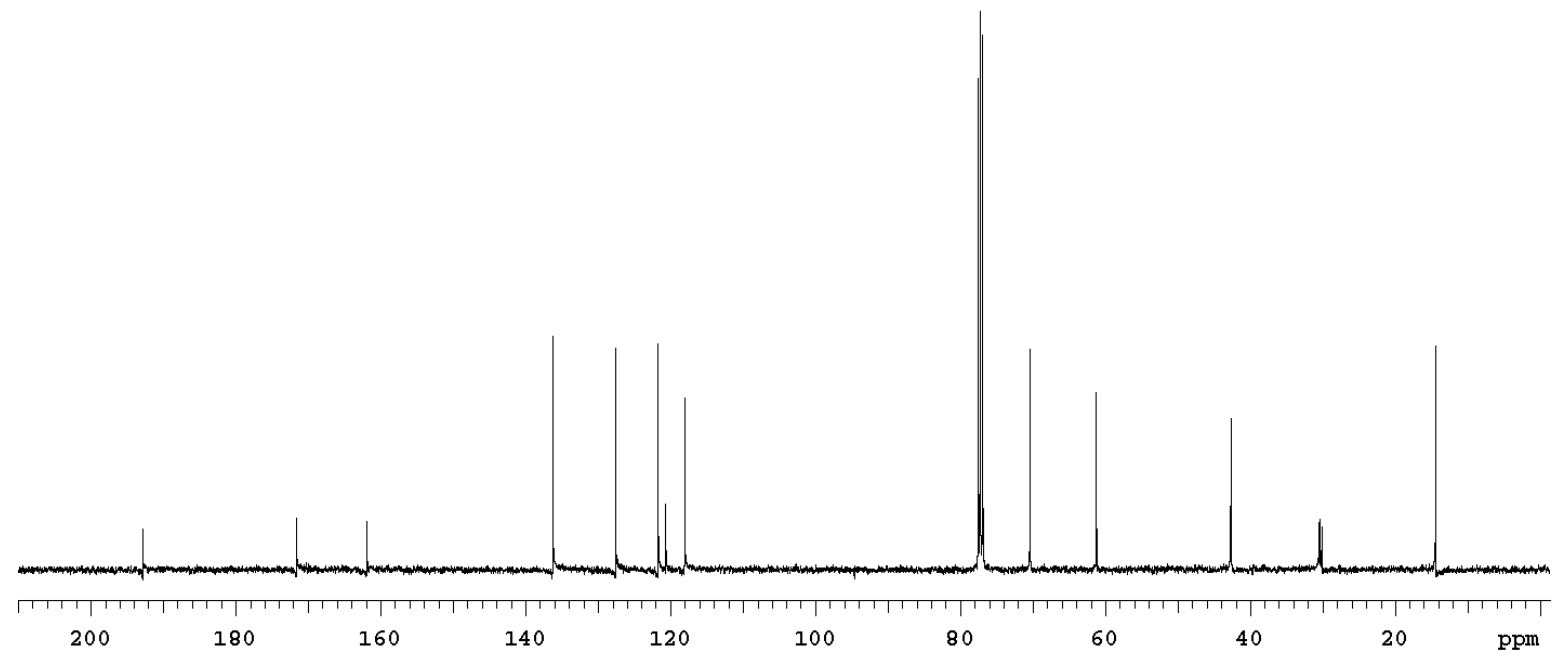




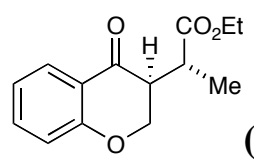

(11)
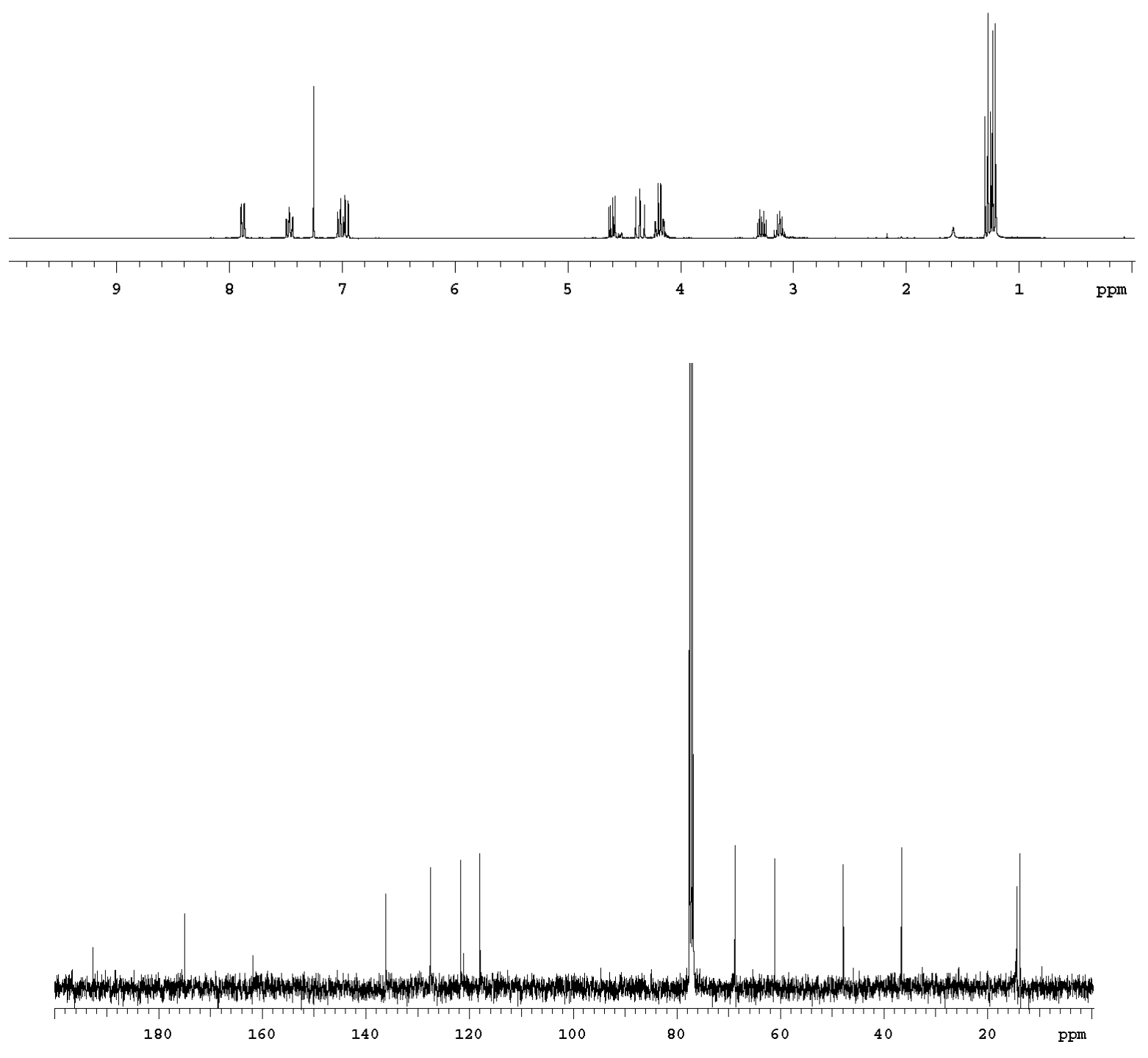
<smiles>CCOC(=O)C(CC)C1COc2ccccc2C1=O</smiles>
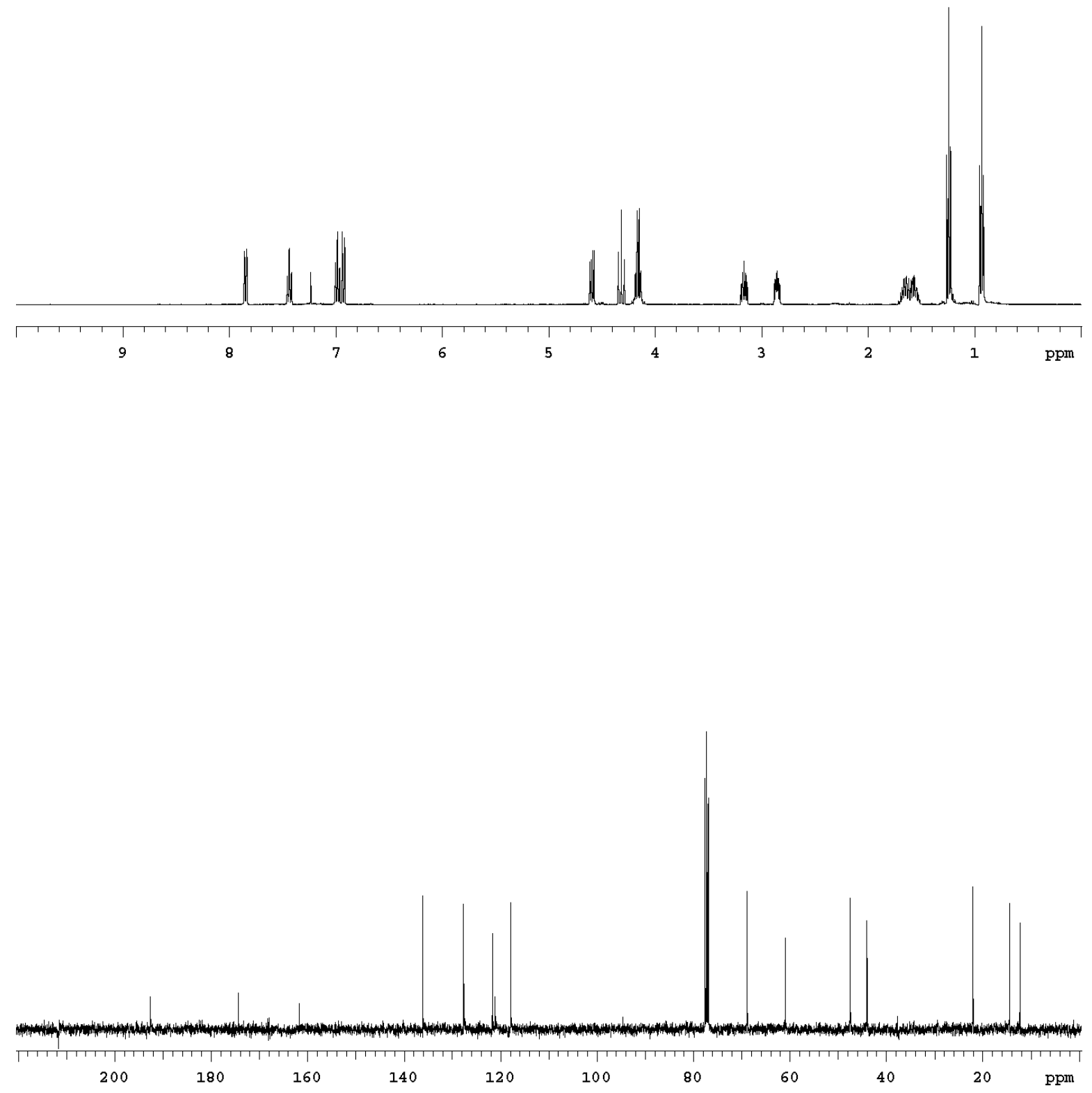
<smiles>CCCCC(OCC)[C@H](C(=O)OCC)[C@H]1COc2ccccc2C1=O</smiles>
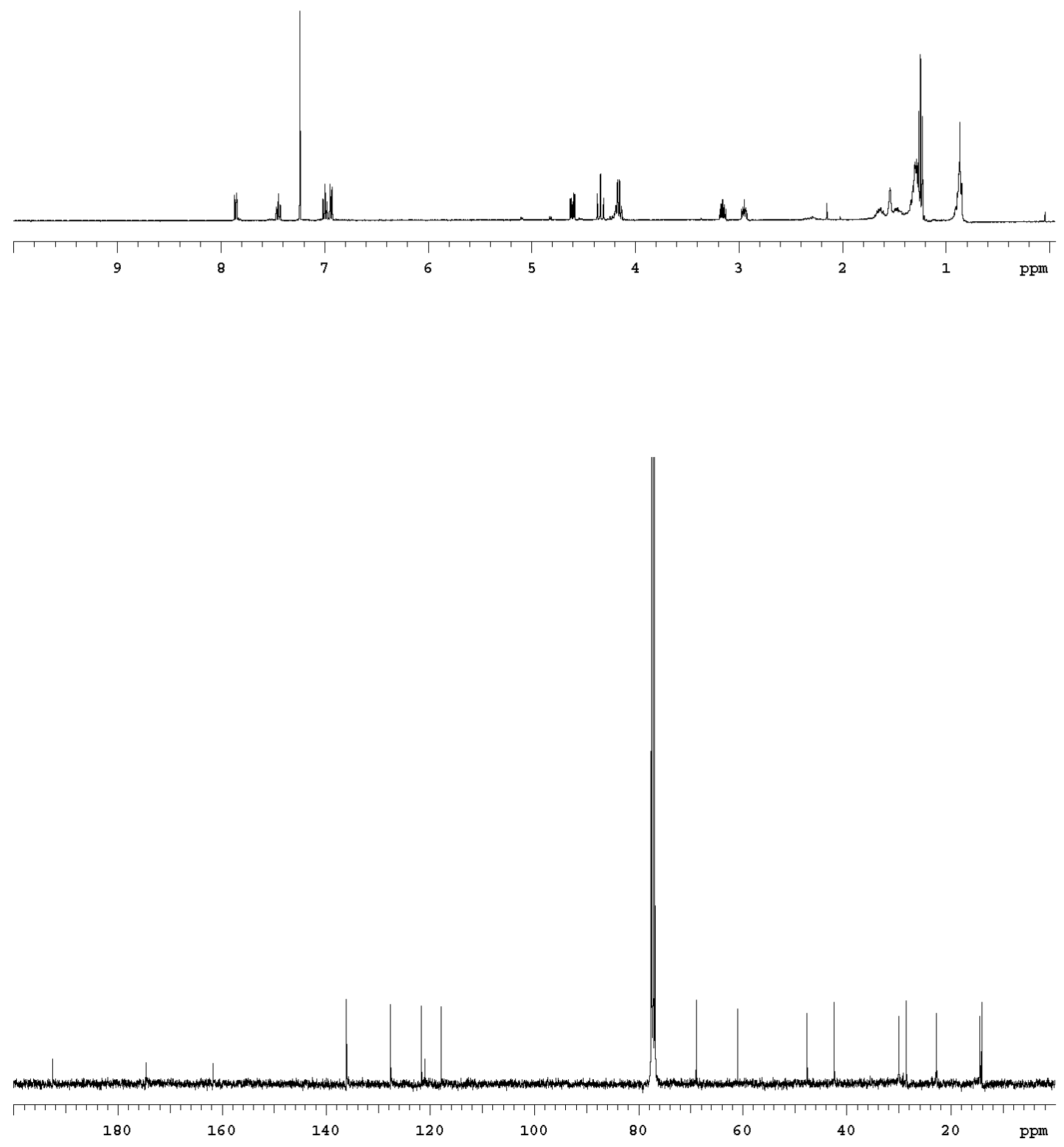
(17)
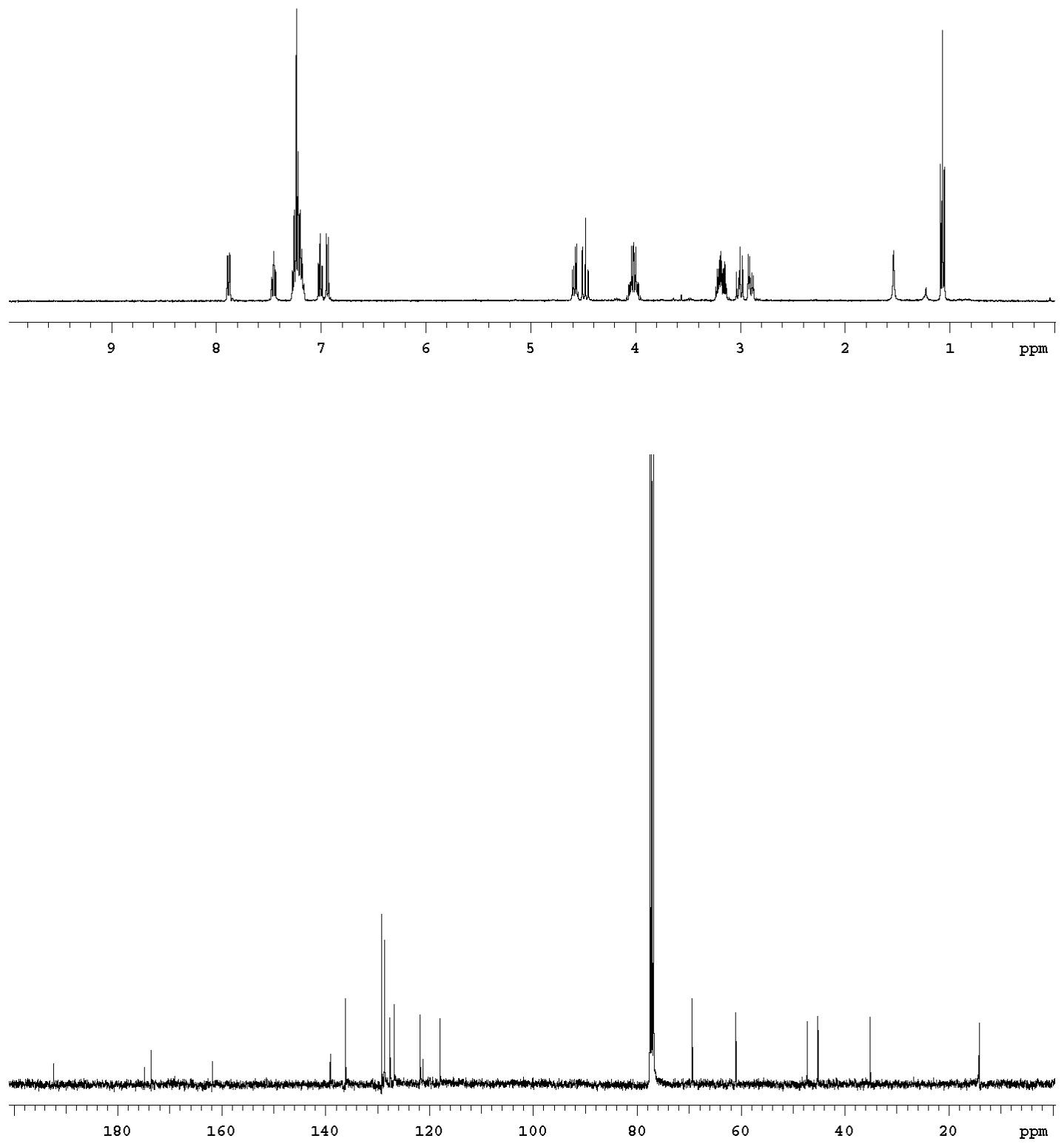

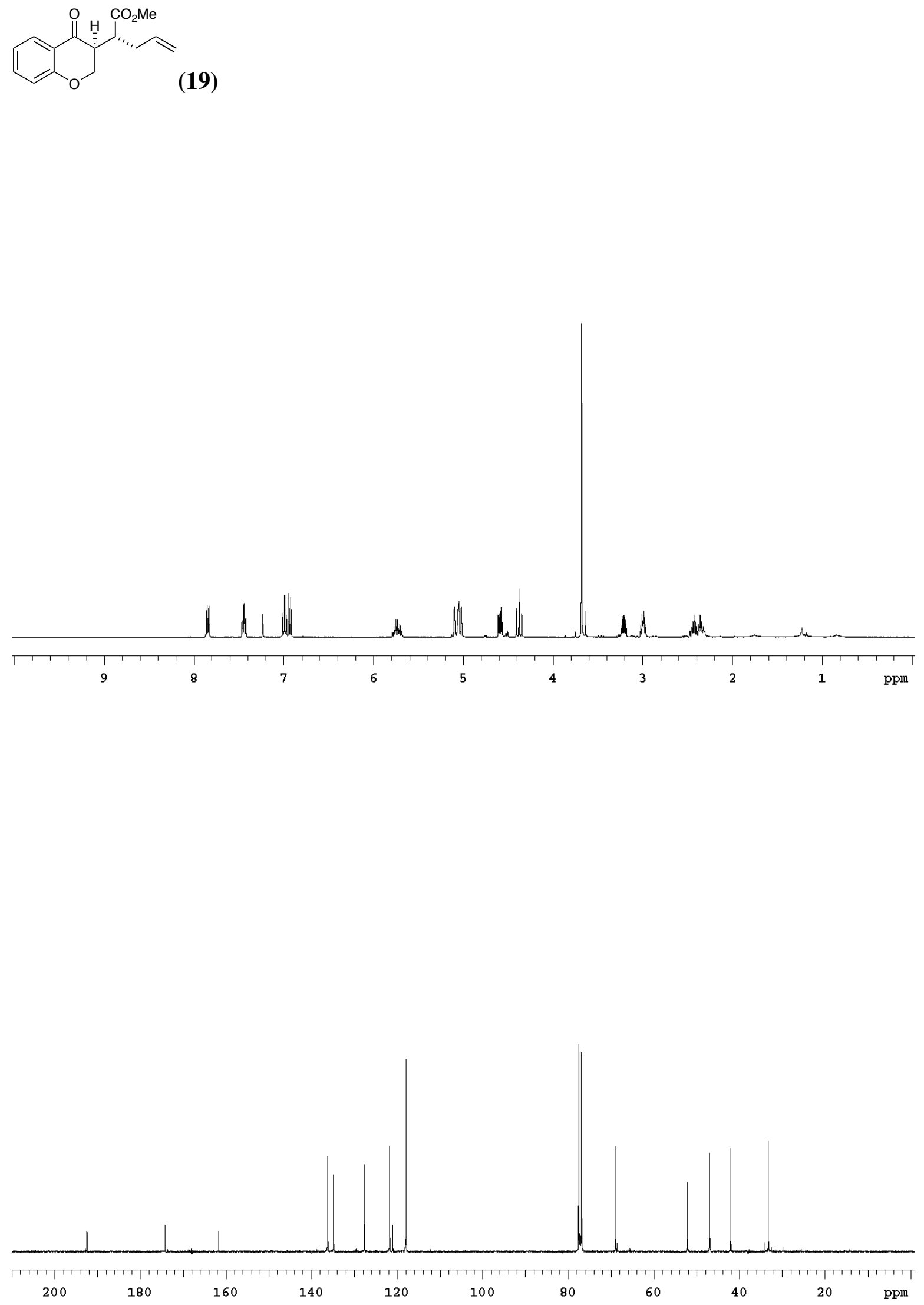
<smiles>CC1([C@@H]2COc3ccccc3C2=O)CCOC1=O</smiles>
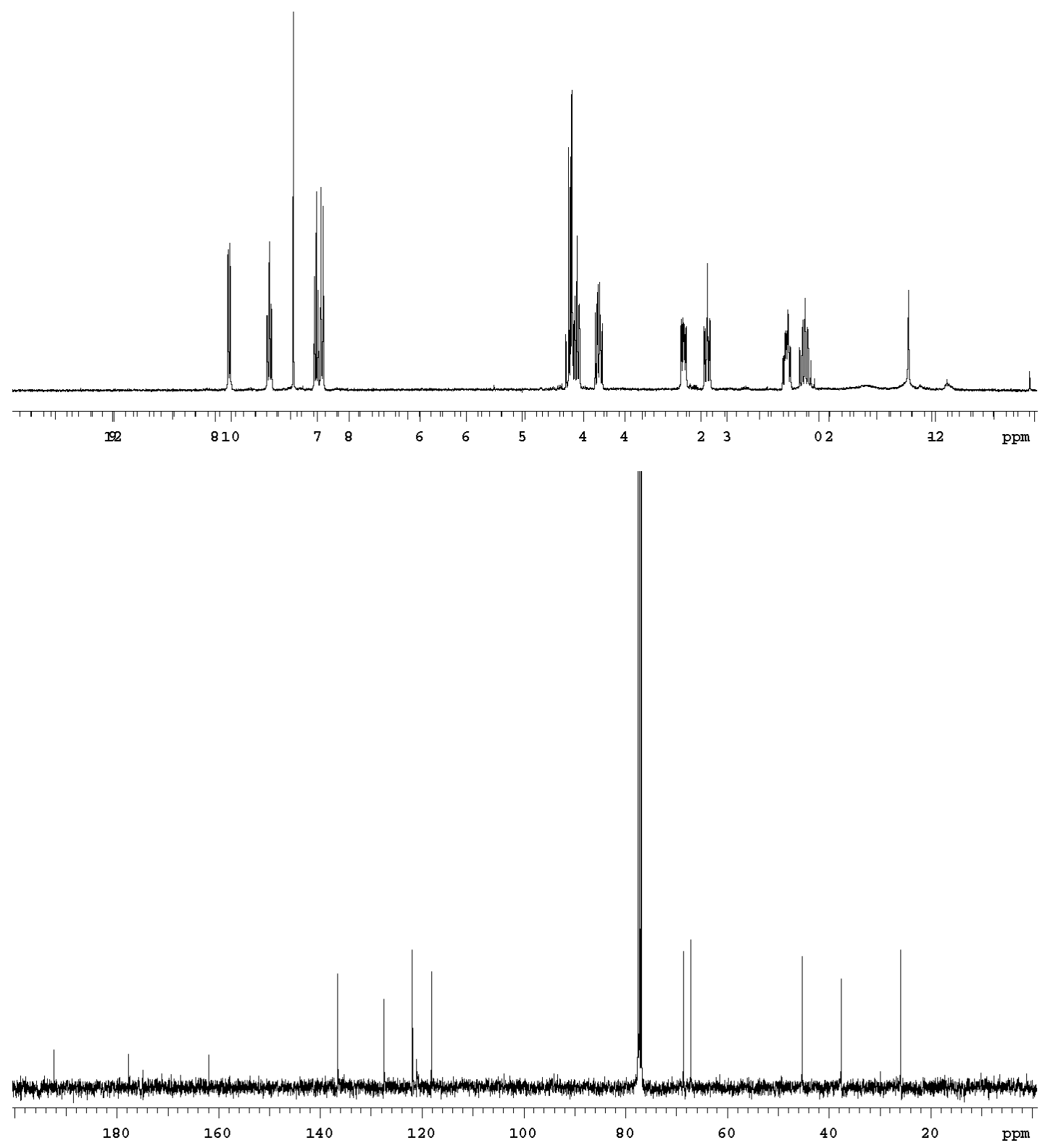

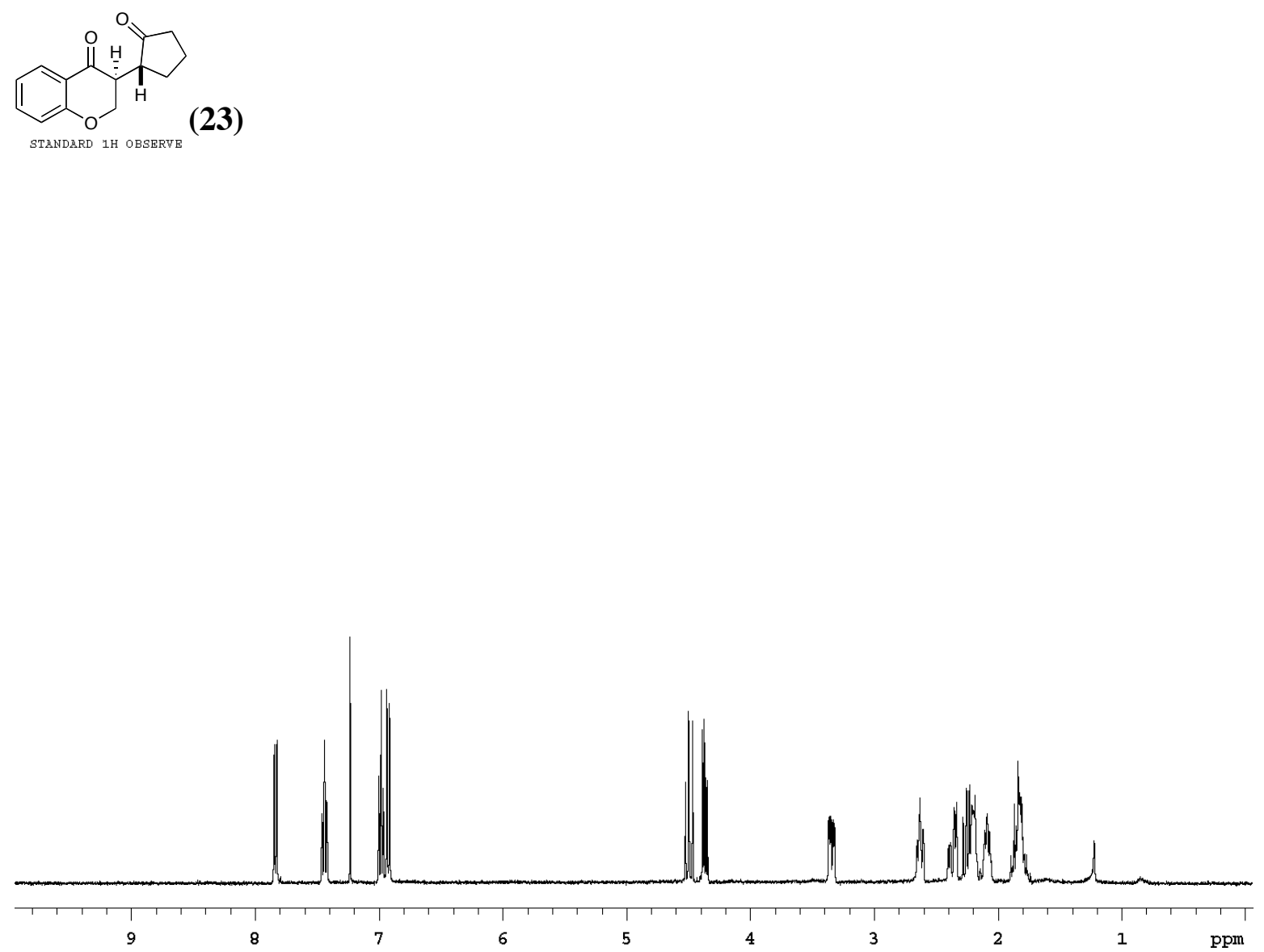

XX-I - 154 - carb on

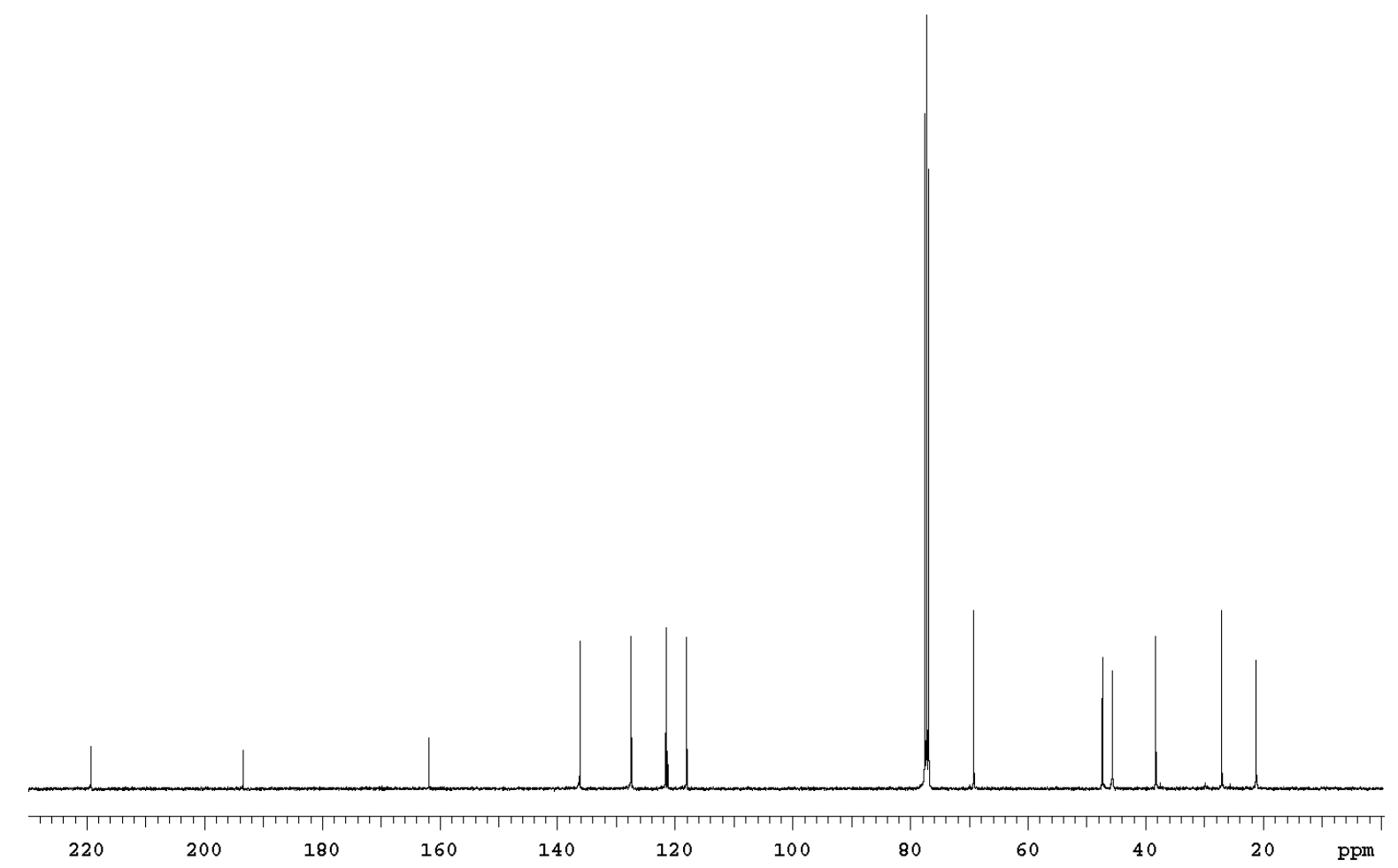




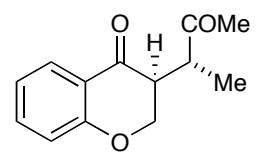

(25)
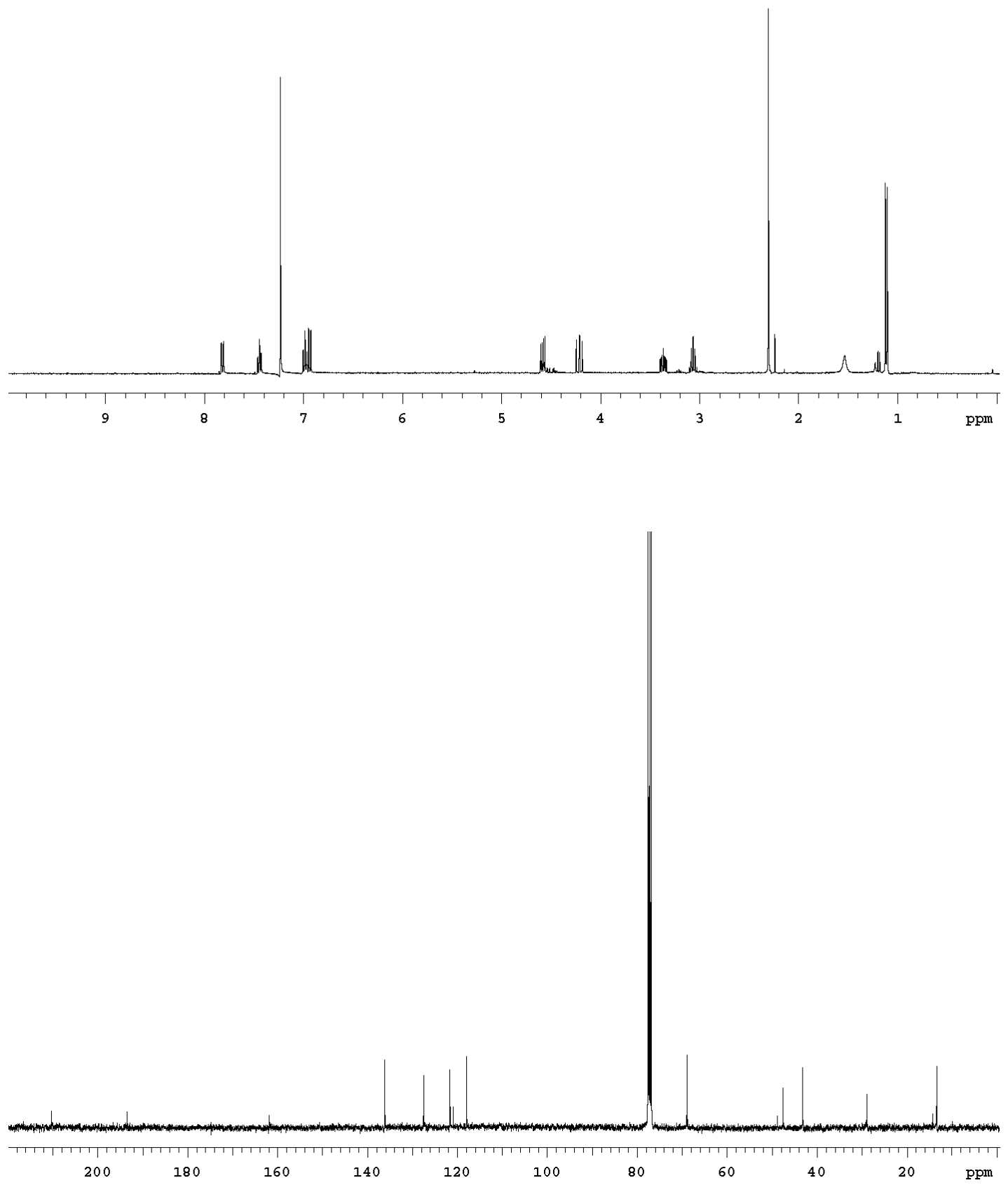

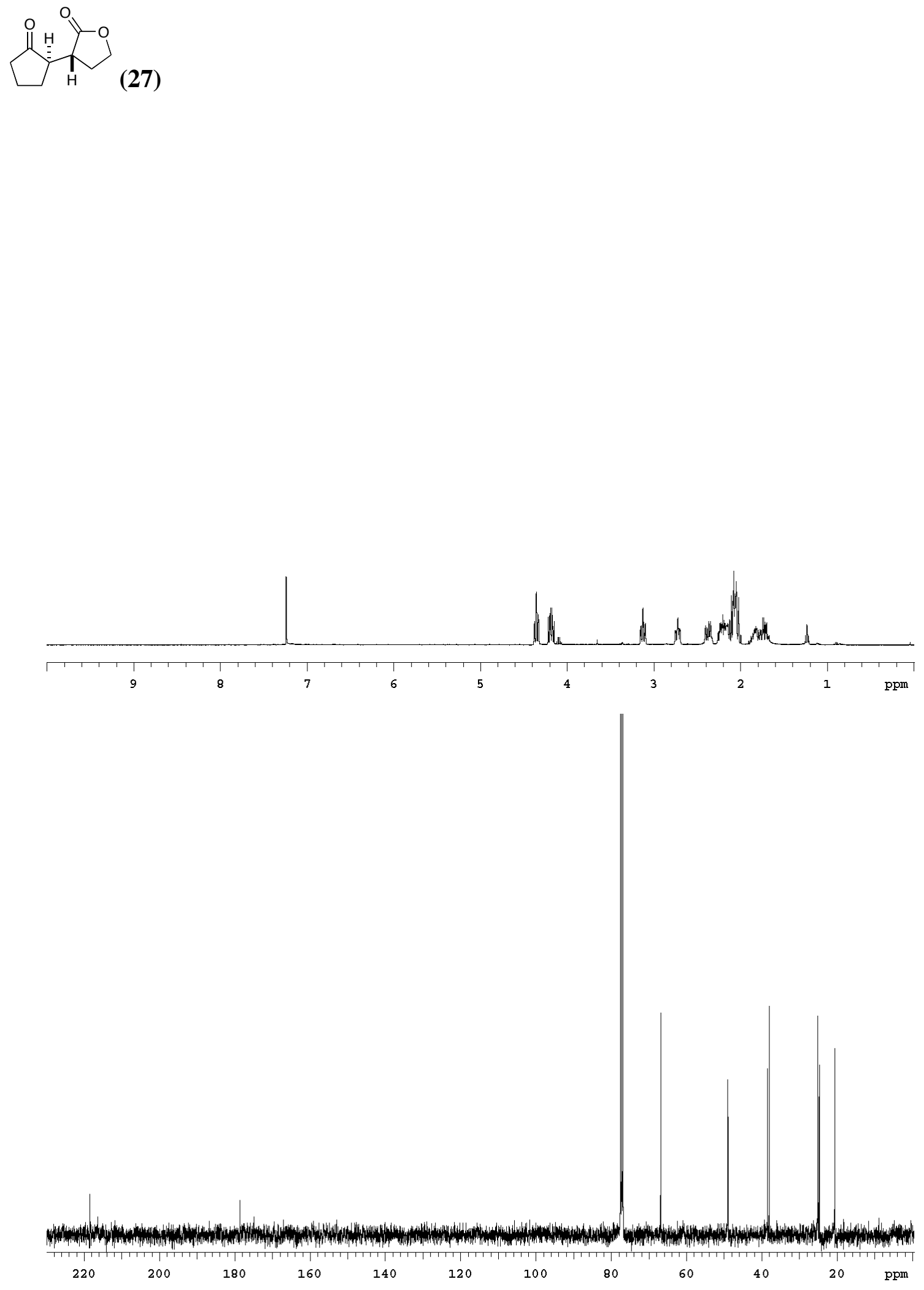

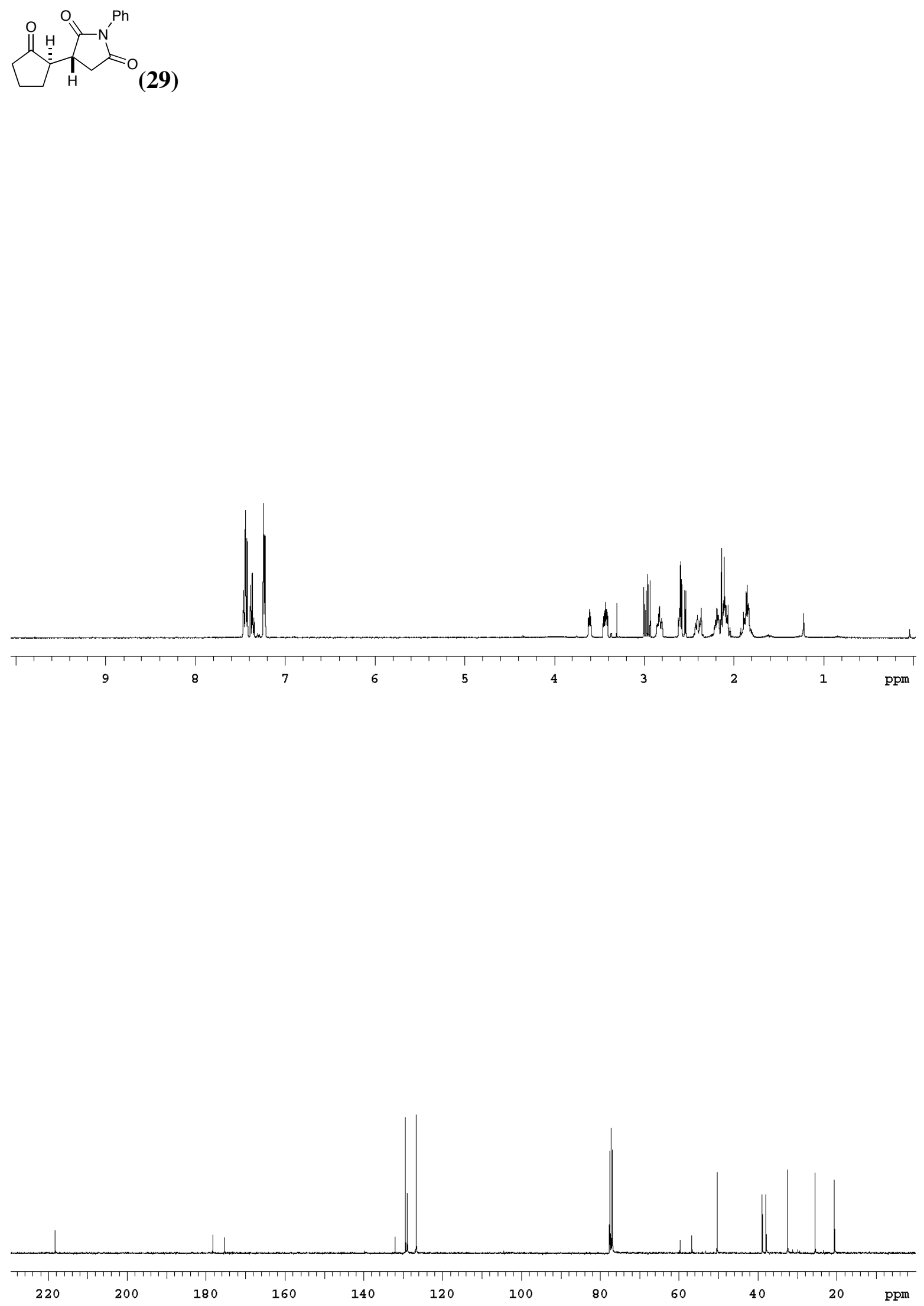


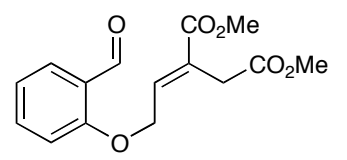

XX-429-E

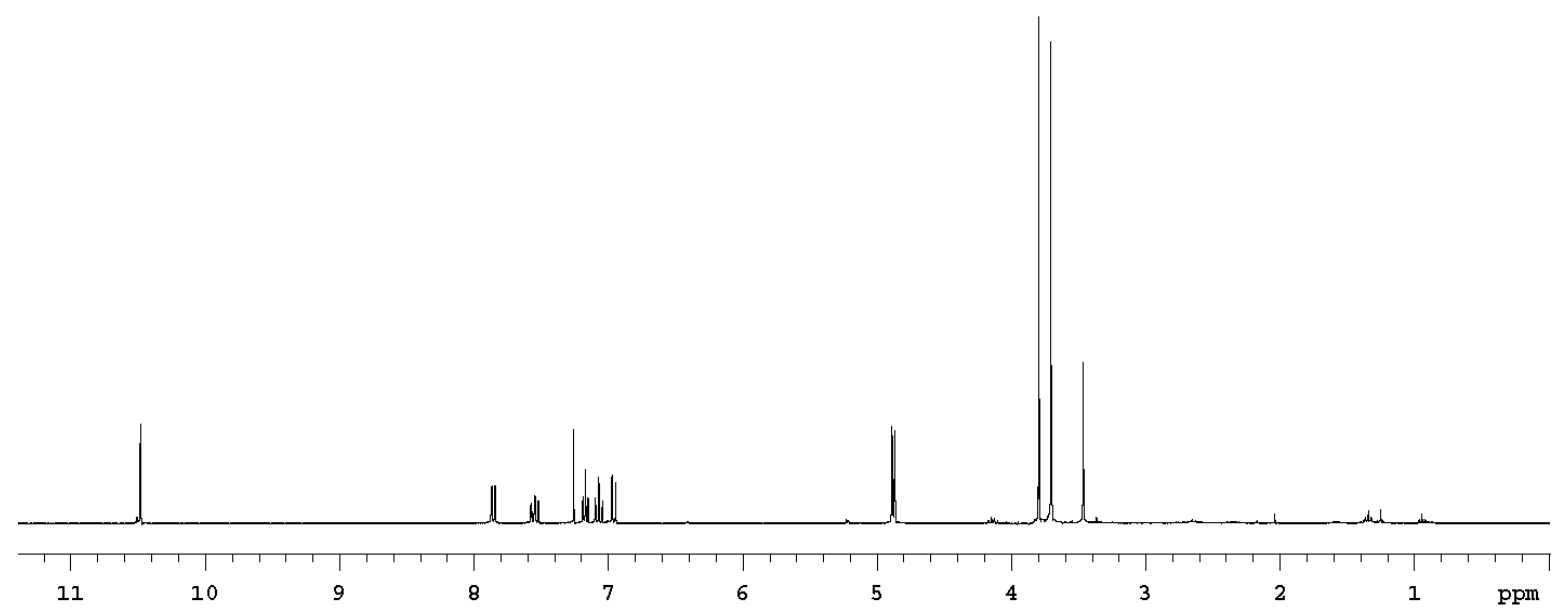



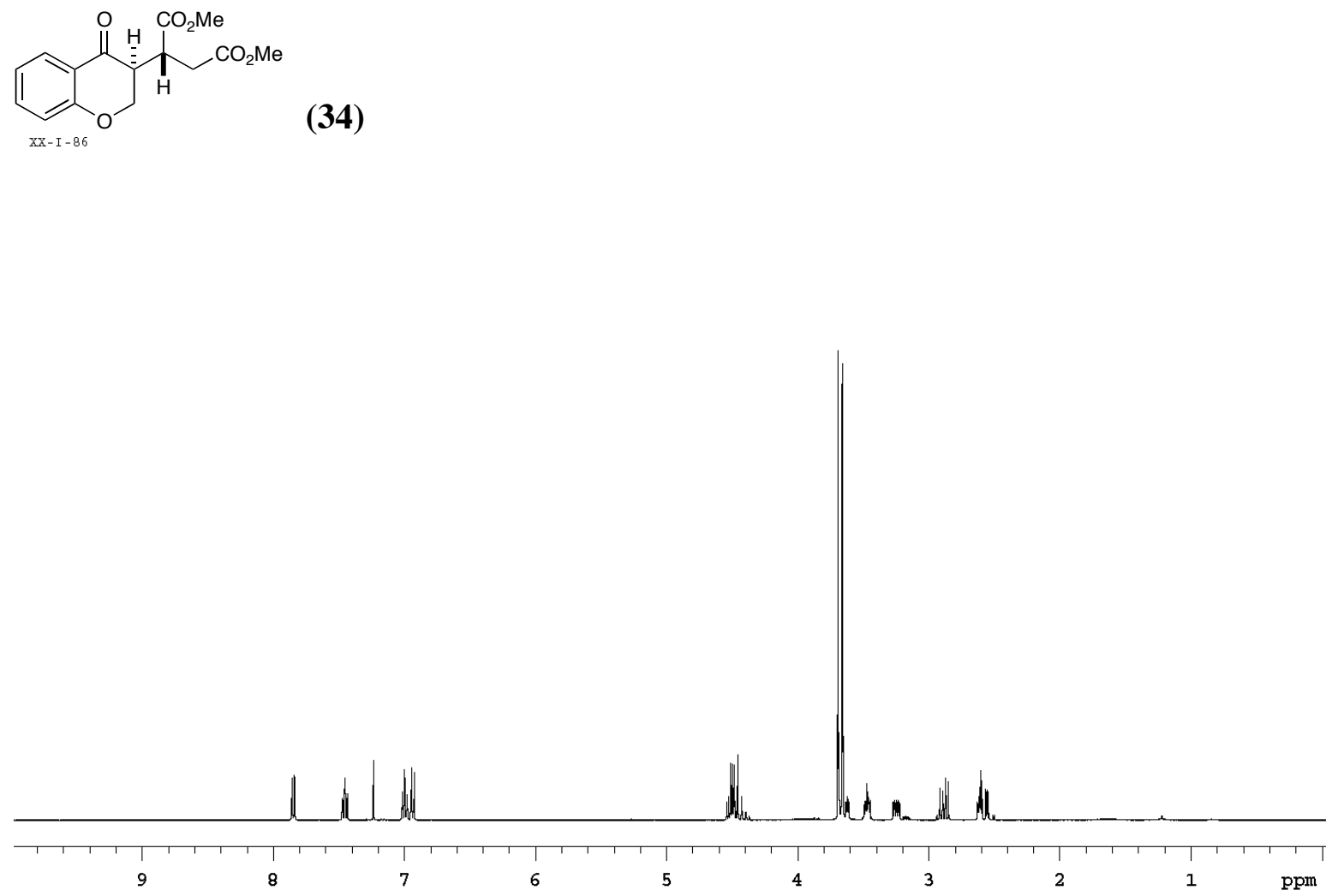

13C OBSERVE

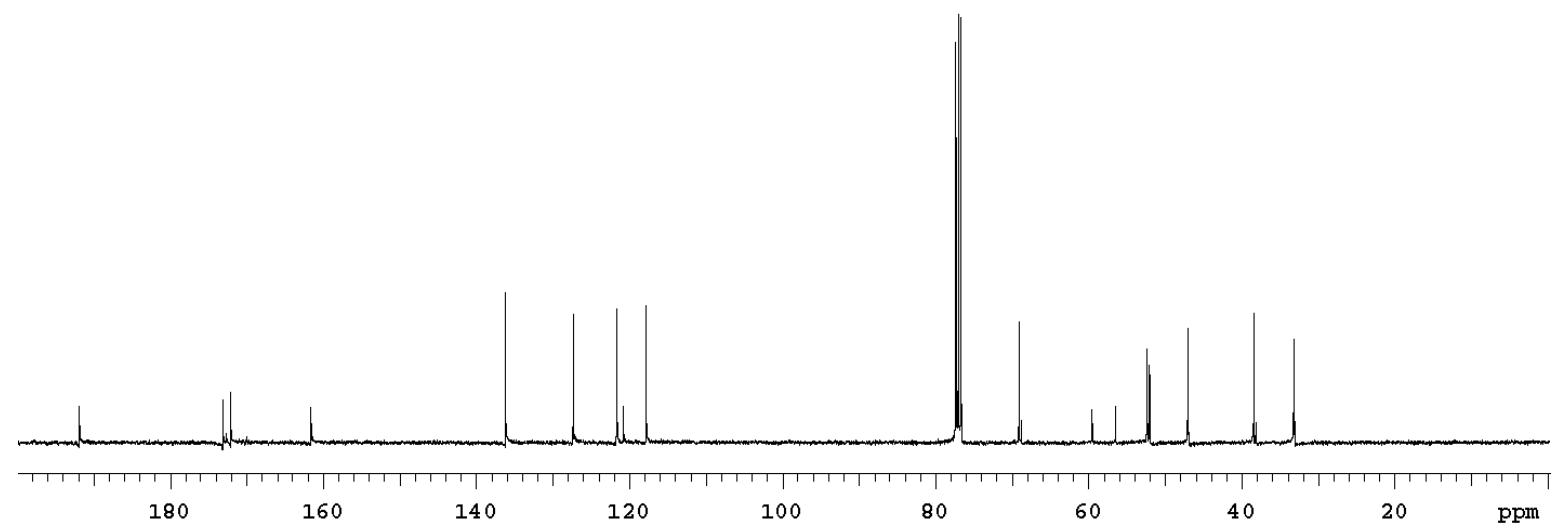



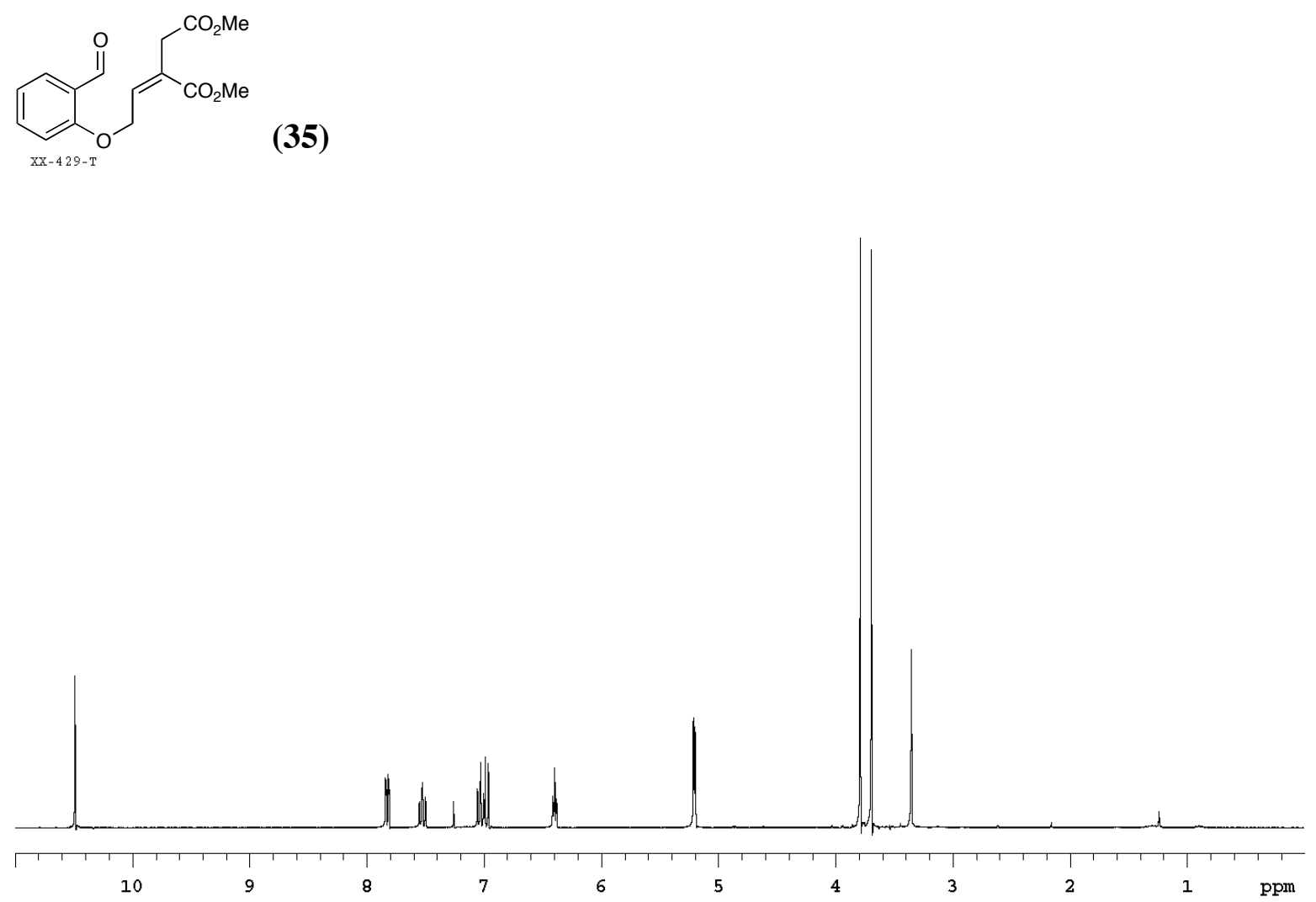

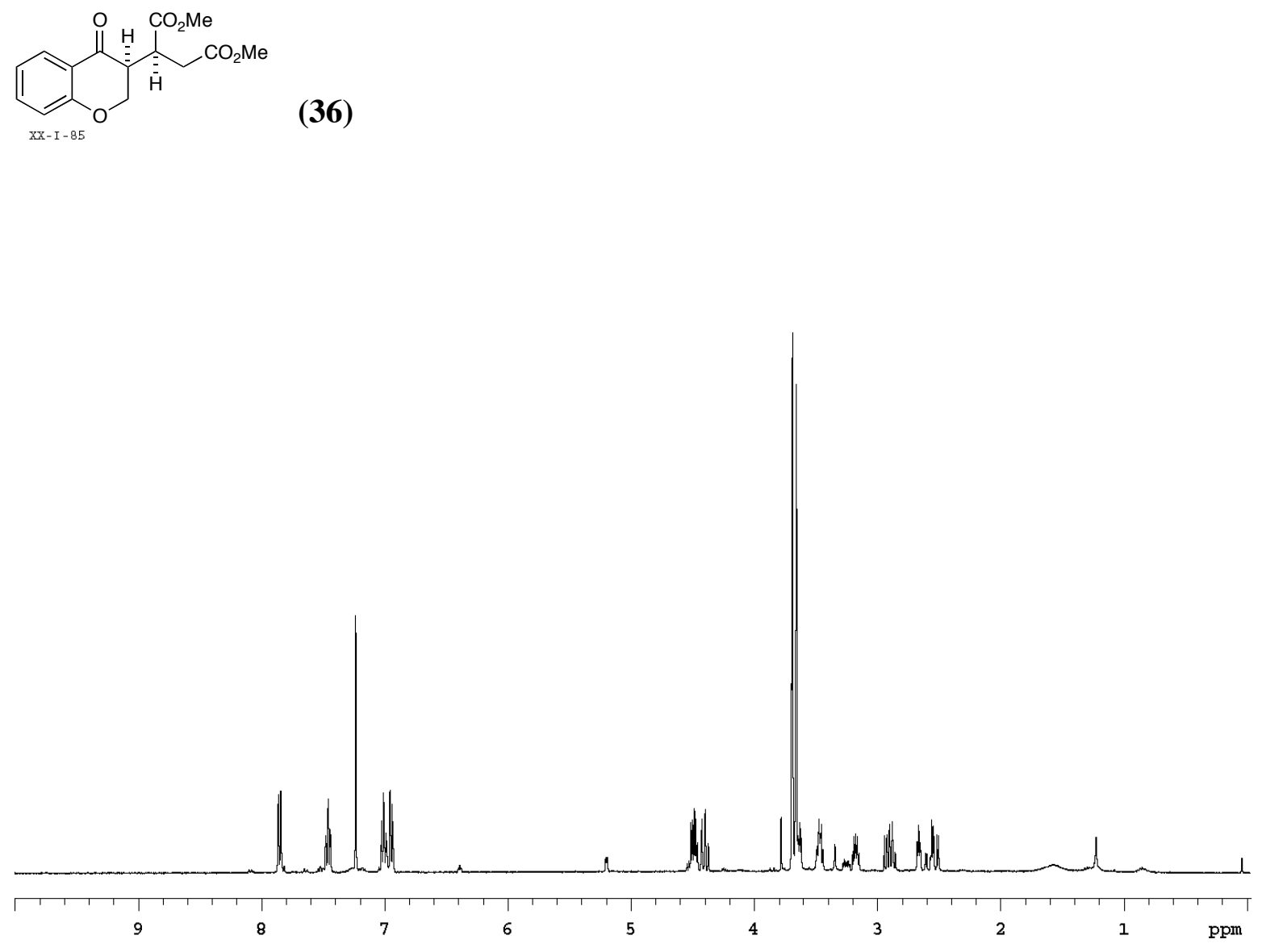

XX-433-carbon

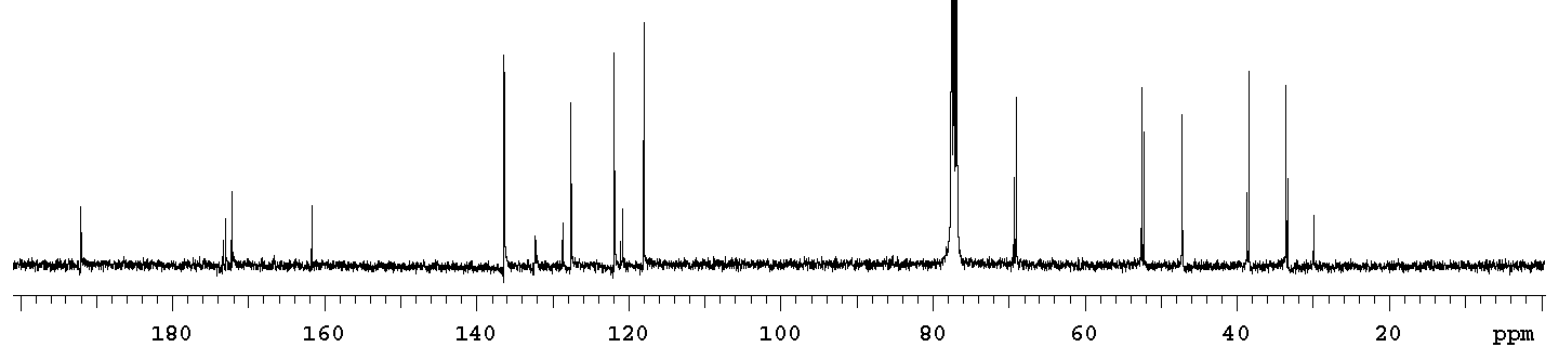




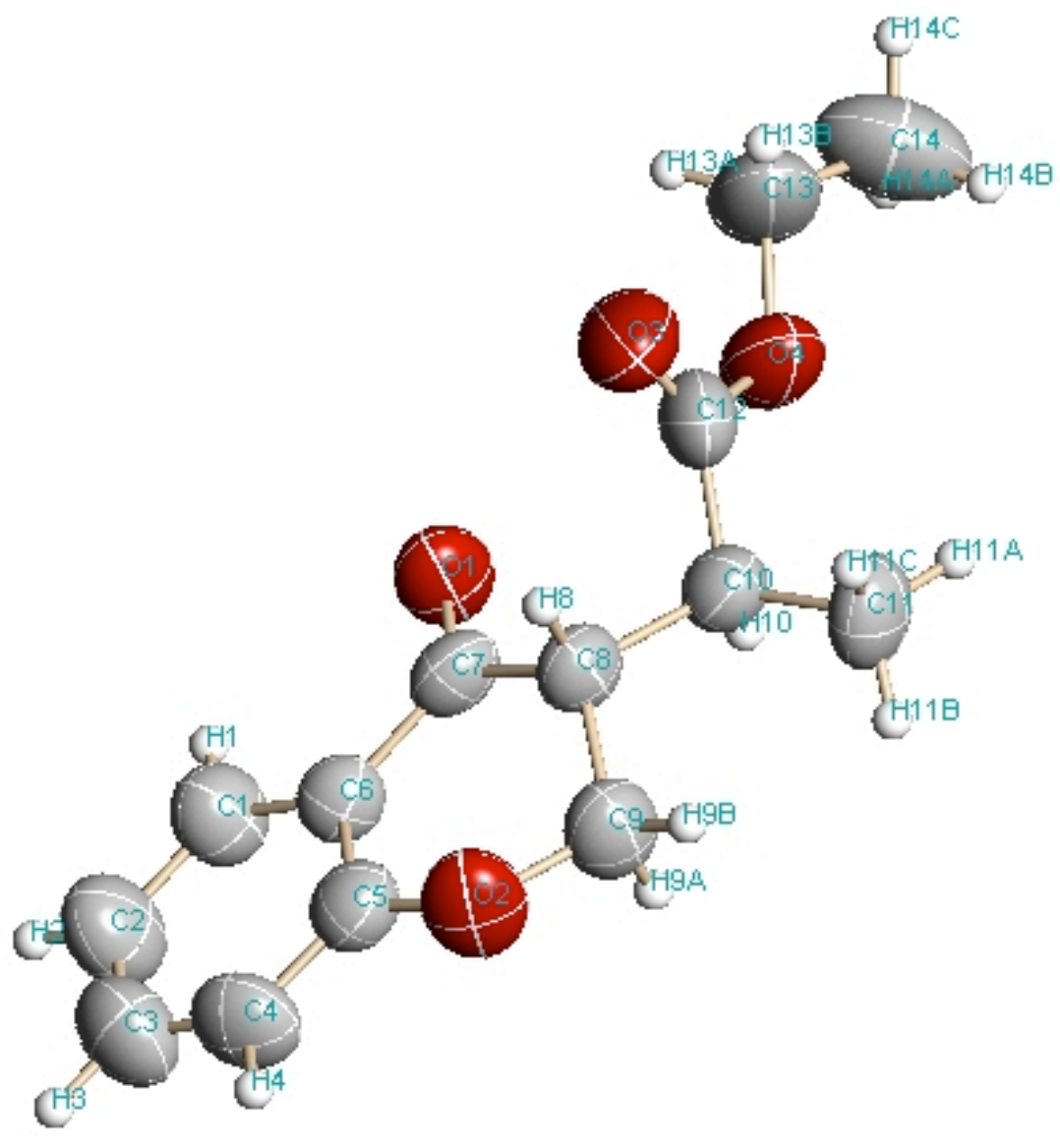

Table 1. Crystal data and structure refinement for try.

Identification code

Empirical formula

Formula weight

Temperature

Wavelength

Crystal system try

C14 H16 O4

248.27

298(2) K

$0.71073 \AA$

Monoclinic 
Space group

Unit cell dimensions

Volume

$\mathrm{Z}$

Density (calculated)

Absorption coefficient

$\mathrm{F}(000)$

Crystal size

Theta range for data collection

Index ranges

Reflections collected

Independent reflections

Completeness to theta $=18.83^{\circ}$

Refinement method

Data / restraints / parameters

Goodness-of-fit on $\mathrm{F}^{2}$

Final $\mathrm{R}$ indices $[\mathrm{I}>2 \operatorname{sigma}(\mathrm{I})]$

$\mathrm{R}$ indices (all data)

Absolute structure parameter

Largest diff. peak and hole
P2(1)

$\mathrm{a}=8.945(18) \AA \quad \alpha=90^{\circ}$.

$\mathrm{b}=6.826(14) \AA \quad \beta=95.07(4)^{\circ}$.

$\mathrm{c}=10.57(2) \AA \quad \gamma=90^{\circ}$.

643(2) $\AA^{3}$

2

$1.283 \mathrm{Mg} / \mathrm{m}^{3}$

$0.094 \mathrm{~mm}^{-1}$

264

$0.08 \times 0.20 \times 0.40 \mathrm{~mm}^{3}$

1.93 to $18.83^{\circ}$.

$-8<=\mathrm{h}<=8,-6<=\mathrm{k}<=6,-9<=\mathrm{l}<=9$

2519

$1015[\mathrm{R}(\mathrm{int})=0.0543]$

$100.0 \%$

Full-matrix least-squares on $\mathrm{F}^{2}$

$1015 / 1 / 165$

1.045

$\mathrm{R} 1=0.0419, \mathrm{wR} 2=0.0980$

$\mathrm{R} 1=0.0531, \mathrm{wR} 2=0.1073$

$0(2)$

0.137 and -0.120 e. $\AA^{-3}$ 
Table 2. Atomic coordinates $\left(\mathrm{x} 10^{4}\right)$ and equivalent isotropic displacement parameters $\left(\AA^{2} \mathrm{x}\right.$ $\left.10^{3}\right)$

for try. $U(e q)$ is defined as one third of the trace of the orthogonalized $U^{i j}$ tensor.

\begin{tabular}{|c|c|c|c|c|}
\hline & $\mathrm{x}$ & $\mathrm{y}$ & z & $\mathrm{U}(\mathrm{eq})$ \\
\hline \multicolumn{5}{|l|}{-} \\
\hline $\mathrm{O}(1)$ & $6875(4)$ & $-1605(5)$ & $5222(3)$ & $83(1)$ \\
\hline $\mathrm{O}(2)$ & $9116(3)$ & $3157(5)$ & $4046(3)$ & $86(1)$ \\
\hline $\mathrm{O}(3)$ & $7555(5)$ & $-61(7)$ & $8126(3)$ & $104(1)$ \\
\hline $\mathrm{O}(4)$ & $5072(5)$ & $-203(6)$ & $7769(3)$ & $98(1)$ \\
\hline $\mathrm{C}(1)$ & $8250(5)$ & $-1783(9)$ & $2900(6)$ & $82(2)$ \\
\hline$C(2)$ & $8983(6)$ & $-1774(10)$ & $1821(6)$ & $97(2)$ \\
\hline$C(3)$ & $9786(6)$ & $-141(13)$ & $1530(6)$ & $97(2)$ \\
\hline$C(4)$ & $9838(6)$ & $1469(10)$ & $2276(6)$ & $85(2)$ \\
\hline$C(5)$ & $9067(6)$ & $1475(8)$ & $3350(5)$ & $68(1)$ \\
\hline$C(6)$ & $8276(5)$ & $-188(8)$ & $3689(5)$ & $59(1)$ \\
\hline$C(7)$ & $7544(5)$ & $-165(8)$ & $4880(4)$ & $63(1)$ \\
\hline $\mathrm{C}(8)$ & $7679(5)$ & $1650(6)$ & $5658(4)$ & $63(1)$ \\
\hline $\mathrm{C}(9)$ & $7877(6)$ & $3387(8)$ & $4810(5)$ & $81(1)$ \\
\hline$C(10)$ & $6328(5)$ & $1873(6)$ & $6455(4)$ & $65(1)$ \\
\hline $\mathrm{C}(11)$ & $6196(6)$ & $3918(7)$ & $7048(5)$ & $85(2)$ \\
\hline$C(12)$ & $6438(7)$ & $421(8)$ & $7518(5)$ & $82(2)$ \\
\hline$C(13)$ & $5001(9)$ & $-1522(11)$ & $8830(6)$ & $144(3)$ \\
\hline$C(14)$ & $3578(8)$ & $-1391(13)$ & $9295(6)$ & $150(3)$ \\
\hline
\end{tabular}


Table 3. Bond lengths $[\AA]$ and angles $\left[{ }^{\circ}\right]$ for try.

\begin{tabular}{|c|c|}
\hline $\mathrm{O}(1)-\mathrm{C}(7)$ & $1.222(6)$ \\
\hline $\mathrm{O}(2)-\mathrm{C}(5)$ & $1.362(6)$ \\
\hline $\mathrm{O}(2)-\mathrm{C}(9)$ & $1.436(6)$ \\
\hline $\mathrm{O}(3)-\mathrm{C}(12)$ & $1.186(6)$ \\
\hline $\mathrm{O}(4)-\mathrm{C}(12)$ & $1.343(6)$ \\
\hline $\mathrm{O}(4)-\mathrm{C}(13)$ & $1.444(7)$ \\
\hline$C(1)-C(2)$ & $1.364(7)$ \\
\hline$C(1)-C(6)$ & $1.370(7)$ \\
\hline$C(2)-C(3)$ & $1.376(9)$ \\
\hline$C(3)-C(4)$ & $1.351(8)$ \\
\hline$C(4)-C(5)$ & $1.379(7)$ \\
\hline$C(5)-C(6)$ & $1.401(7)$ \\
\hline$C(6)-C(7)$ & $1.469(7)$ \\
\hline$C(7)-C(8)$ & $1.486(6)$ \\
\hline$C(8)-C(9)$ & $1.506(7)$ \\
\hline$C(8)-C(10)$ & $1.540(6)$ \\
\hline$C(10)-C(12)$ & $1.494(7)$ \\
\hline$C(10)-C(11)$ & $1.539(7)$ \\
\hline$C(13)-C(14)$ & $1.407(8)$ \\
\hline $\mathrm{C}(5)-\mathrm{O}(2)-\mathrm{C}(9)$ & $114.0(4)$ \\
\hline $\mathrm{C}(12)-\mathrm{O}(4)-\mathrm{C}(13)$ & $117.1(4)$ \\
\hline $\mathrm{C}(2)-\mathrm{C}(1)-\mathrm{C}(6)$ & $121.4(5)$ \\
\hline$C(1)-C(2)-C(3)$ & $119.3(5)$ \\
\hline$C(4)-C(3)-C(2)$ & $121.4(5)$ \\
\hline$C(3)-C(4)-C(5)$ & $119.2(5)$ \\
\hline $\mathrm{O}(2)-\mathrm{C}(5)-\mathrm{C}(4)$ & $116.9(6)$ \\
\hline $\mathrm{O}(2)-\mathrm{C}(5)-\mathrm{C}(6)$ & $122.5(5)$ \\
\hline$C(4)-C(5)-C(6)$ & $120.6(5)$ \\
\hline$C(1)-C(6)-C(5)$ & $118.0(4)$ \\
\hline$C(1)-C(6)-C(7)$ & $123.0(5)$ \\
\hline$C(5)-C(6)-C(7)$ & $119.0(5)$ \\
\hline $\mathrm{O}(1)-\mathrm{C}(7)-\mathrm{C}(6)$ & $120.8(5)$ \\
\hline $\mathrm{O}(1)-\mathrm{C}(7)-\mathrm{C}(8)$ & $121.7(4)$ \\
\hline
\end{tabular}




$\begin{array}{ll}\mathrm{C}(6)-\mathrm{C}(7)-\mathrm{C}(8) & 117.5(5) \\ \mathrm{C}(7)-\mathrm{C}(8)-\mathrm{C}(9) & 109.6(4) \\ \mathrm{C}(7)-\mathrm{C}(8)-\mathrm{C}(10) & 110.8(4) \\ \mathrm{C}(9)-\mathrm{C}(8)-\mathrm{C}(10) & 112.8(4) \\ \mathrm{O}(2)-\mathrm{C}(9)-\mathrm{C}(8) & 112.7(4) \\ \mathrm{C}(12)-\mathrm{C}(10)-\mathrm{C}(11) & 107.3(4) \\ \mathrm{C}(12)-\mathrm{C}(10)-\mathrm{C}(8) & 110.1(4) \\ \mathrm{C}(11)-\mathrm{C}(10)-\mathrm{C}(8) & 113.8(4) \\ \mathrm{O}(3)-\mathrm{C}(12)-\mathrm{O}(4) & 122.9(5) \\ \mathrm{O}(3)-\mathrm{C}(12)-\mathrm{C}(10) & 126.0(6) \\ \mathrm{O}(4)-\mathrm{C}(12)-\mathrm{C}(10) & 111.0(5) \\ \mathrm{C}(14)-\mathrm{C}(13)-\mathrm{O}(4) & 109.6(6)\end{array}$

Symmetry transformations used to generate equivalent atoms: 
Table 4. Anisotropic displacement parameters $\left(\AA^{2} \times 10^{3}\right)$ for try. The anisotropic displacement factor exponent takes the form: $-2 \pi^{2}\left[h^{2} a^{* 2} U^{11}+\ldots+2 h k a^{*} b^{*} U^{12}\right]$

\begin{tabular}{lcccccc}
\hline & $\mathrm{U}^{11}$ & $\mathrm{U}^{22}$ & $\mathrm{U}^{33}$ & $\mathrm{U}^{23}$ & $\mathrm{U}^{13}$ & $\mathrm{U}^{12}$ \\
\hline $\mathrm{O}(1)$ & $100(2)$ & $55(2)$ & $95(2)$ & $2(2)$ & $26(2)$ & $-11(2)$ \\
$\mathrm{O}(2)$ & $84(3)$ & $69(3)$ & $107(3)$ & $-1(2)$ & $27(2)$ & $-23(2)$ \\
$\mathrm{O}(3)$ & $106(3)$ & $116(3)$ & $89(2)$ & $13(2)$ & $7(2)$ & $37(3)$ \\
$\mathrm{O}(4)$ & $99(3)$ & $86(3)$ & $115(3)$ & $36(2)$ & $35(2)$ & $16(2)$ \\
$\mathrm{C}(1)$ & $95(4)$ & $68(4)$ & $83(4)$ & $-9(4)$ & $16(3)$ & $-7(3)$ \\
$\mathrm{C}(2)$ & $120(5)$ & $87(4)$ & $86(4)$ & $-19(4)$ & $25(4)$ & $-10(5)$ \\
$\mathrm{C}(3)$ & $87(4)$ & $117(5)$ & $90(4)$ & $-4(5)$ & $28(3)$ & $-1(4)$ \\
$\mathrm{C}(4)$ & $75(4)$ & $96(6)$ & $87(4)$ & $14(4)$ & $23(3)$ & $-14(3)$ \\
$\mathrm{C}(5)$ & $63(3)$ & $66(5)$ & $76(4)$ & $5(4)$ & $4(3)$ & $5(3)$ \\
$\mathrm{C}(6)$ & $56(3)$ & $52(3)$ & $69(3)$ & $1(4)$ & $6(2)$ & $-7(3)$ \\
$\mathrm{C}(7)$ & $64(3)$ & $46(3)$ & $78(4)$ & $10(3)$ & $3(3)$ & $1(3)$ \\
$\mathrm{C}(8)$ & $74(4)$ & $45(3)$ & $72(3)$ & $-1(3)$ & $9(3)$ & $0(3)$ \\
$\mathrm{C}(9)$ & $93(4)$ & $60(3)$ & $94(3)$ & $1(3)$ & $23(3)$ & $0(3)$ \\
$\mathrm{C}(10)$ & $62(3)$ & $63(4)$ & $72(3)$ & $8(3)$ & $8(3)$ & $7(3)$ \\
$\mathrm{C}(11)$ & $90(4)$ & $57(4)$ & $110(4)$ & $-13(3)$ & $20(3)$ & $11(3)$ \\
$\mathrm{C}(12)$ & $80(5)$ & $88(5)$ & $81(4)$ & $3(4)$ & $25(3)$ & $27(4)$ \\
$\mathrm{C}(13)$ & $187(7)$ & $110(5)$ & $147(5)$ & $66(5)$ & $87(5)$ & $48(5)$ \\
$\mathrm{C}(14)$ & $172(7)$ & $171(7)$ & $111(5)$ & $35(5)$ & $37(5)$ & $-38(6)$ \\
& & & & & & \\
\hline
\end{tabular}


Table 5. Hydrogen coordinates $\left(\mathrm{x} 10^{4}\right)$ and isotropic displacement parameters $\left(\AA^{2} \times 10^{3}\right)$ for try.

\begin{tabular}{|c|c|c|c|c|}
\hline & $\mathrm{x}$ & $\mathrm{y}$ & $\mathrm{z}$ & $\mathrm{U}(\mathrm{eq})$ \\
\hline \multicolumn{5}{|l|}{-} \\
\hline $\mathrm{H}(1)$ & 7721 & -2895 & 3104 & 98 \\
\hline $\mathrm{H}(2)$ & 8939 & -2861 & 1288 & 116 \\
\hline $\mathrm{H}(3)$ & 10304 & -146 & 805 & 116 \\
\hline $\mathrm{H}(4)$ & 10387 & 2561 & 2067 & 102 \\
\hline $\mathrm{H}(8)$ & 8585 & 1535 & 6246 & 76 \\
\hline $\mathrm{H}(9 \mathrm{~A})$ & 6964 & 3577 & 4257 & 98 \\
\hline $\mathrm{H}(9 \mathrm{~B})$ & 8036 & 4551 & 5331 & 98 \\
\hline $\mathrm{H}(10)$ & 5408 & 1607 & 5908 & 79 \\
\hline $\mathrm{H}(11 \mathrm{~A})$ & 5337 & 3953 & 7531 & 128 \\
\hline $\mathrm{H}(11 \mathrm{~B})$ & 6084 & 4883 & 6386 & 128 \\
\hline $\mathrm{H}(11 \mathrm{C})$ & 7085 & 4192 & 7596 & 128 \\
\hline $\mathrm{H}(13 \mathrm{~A})$ & 5178 & -2855 & 8562 & 172 \\
\hline $\mathrm{H}(13 \mathrm{~B})$ & 5772 & -1183 & 9498 & 172 \\
\hline $\mathrm{H}(14 \mathrm{~A})$ & 2831 & -1893 & 8671 & 225 \\
\hline $\mathrm{H}(14 \mathrm{~B})$ & 3360 & -46 & 9470 & 225 \\
\hline $\mathrm{H}(14 \mathrm{C})$ & 3571 & -2145 & 10062 & 225 \\
\hline
\end{tabular}




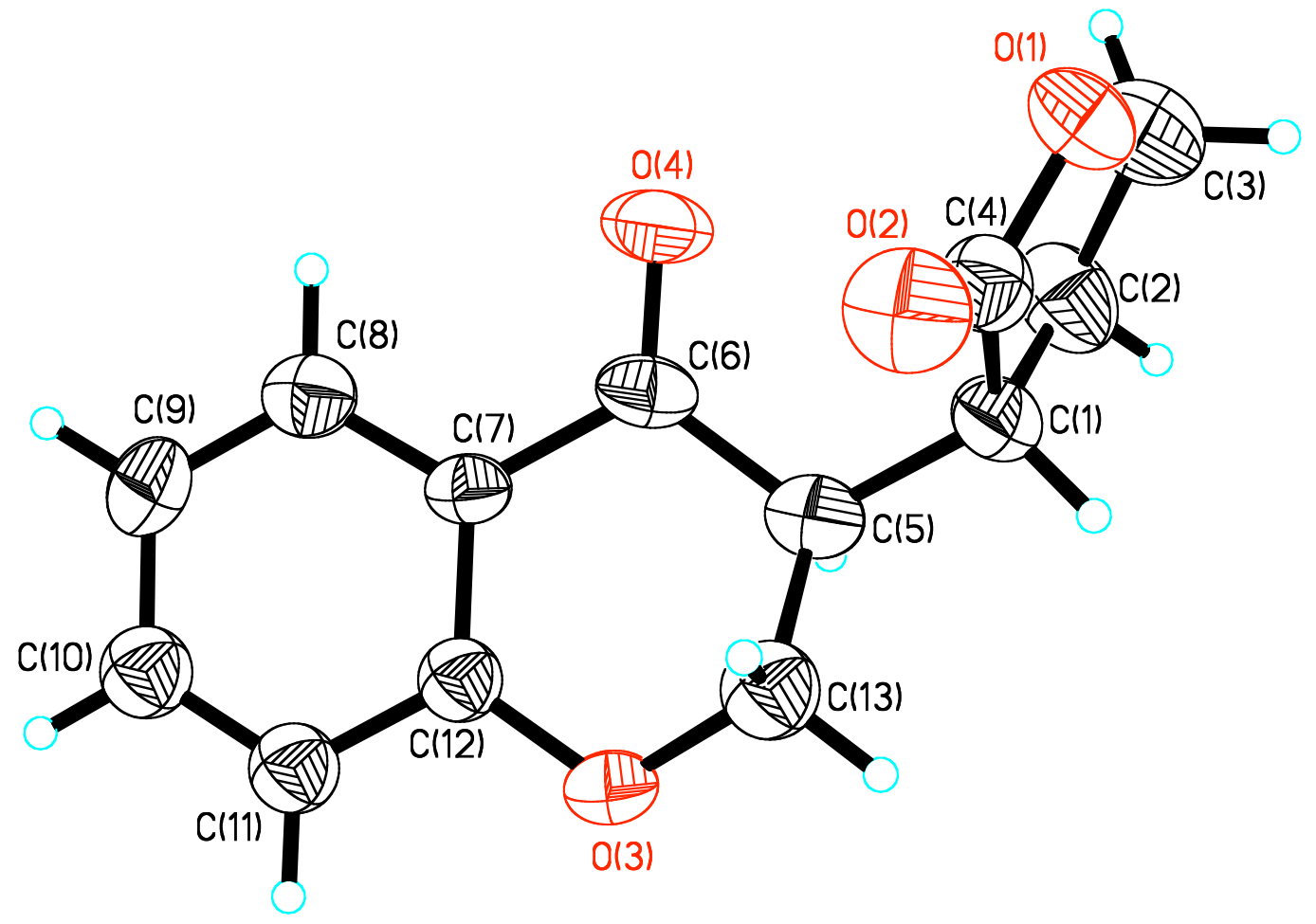

Table 1. Crystal data and structure refinement for Rovis7.

Identification code

Empirical formula

Formula weight

Temperature

Wavelength

Crystal system

Space group

Unit cell dimensions

Volume

Z
ROVIS7

C13 $\mathrm{H} 12 \mathrm{O} 4$

232.23

298(2) K

$0.71073 \AA$

Orthorhombic

$\mathrm{P} 2(1) 2(1) 2(1)$

$\mathrm{a}=6.4729(10) \AA$

$\alpha=90^{\circ}$.

$\mathrm{b}=8.1736(13) \AA$

$\beta=90^{\circ}$.

$\mathrm{c}=21.179(3) \AA$

$\gamma=90^{\circ}$.

1120.5(3) $\AA^{3}$

4 


\author{
Density (calculated) \\ Absorption coefficient \\ Absorption correction \\ $\mathrm{F}(000)$ \\ Crystal size \\ Theta range for data collection \\ *High-resolution data truncated at $1.0 \AA$ \\ Index ranges \\ Reflections collected \\ Independent reflections \\ Completeness to theta $=20.81^{\circ}$ \\ Refinement method \\ Data / restraints / parameters \\ Goodness-of-fit on $\mathrm{F}^{2}$ \\ Final $\mathrm{R}$ indices [I $>2 \operatorname{sigma}(\mathrm{I})]$ \\ $\mathrm{R}$ indices (all data) \\ Largest diff. peak and hole
}

$1.377 \mathrm{Mg} / \mathrm{m}^{3}$

$0.102 \mathrm{~mm}^{-1}$

\section{SADABS}

488

$0.40 \times 0.38 \times 0.35 \mathrm{~mm}^{3}$

1.92 to $20.81^{\circ}$.

$-6<=\mathrm{h}<=6,-8<=\mathrm{k}<=8,-21<=\mathrm{l}<=21$

5636

$1179[\mathrm{R}(\mathrm{int})=0.0331]$

$100.0 \%$

Full-matrix least-squares on $\mathrm{F}^{2}$

1179 / 0 / 134

1.016

$\mathrm{R} 1=0.0446,{ }_{\mathrm{w}} 2=0.1066$

$\mathrm{R} 1=0.0603, \mathrm{wR}_{2}=0.1162$

0.191 and -0.118 e. $\AA^{-3}$ 
Table 2. Atomic coordinates ( $\left.\times 10^{4}\right)$ and equivalent isotropic displacement parameters $\left(\AA^{2} \times 10^{3}\right)$ for rovis7. $U(e q)$ is defined as one third of the trace of the orthogonalized $U^{i j}$ tensor.

\begin{tabular}{lcccc}
\hline & $\mathrm{x}$ & $\mathrm{y}$ & $\mathrm{z}$ & $\mathrm{U}(\mathrm{eq})$ \\
\hline $\mathrm{O}(1)$ & $2022(5)$ & $4053(4)$ & $7368(1)$ & $90(1)$ \\
$\mathrm{O}(2)$ & $3655(6)$ & $5720(5)$ & $8006(2)$ & $104(1)$ \\
$\mathrm{O}(3)$ & $4123(4)$ & $4004(3)$ & $10027(1)$ & $78(1)$ \\
$\mathrm{O}(4)$ & $-487(5)$ & $4500(4)$ & $8735(1)$ & $98(1)$ \\
$\mathrm{C}(1)$ & $3127(8)$ & $2874(6)$ & $8308(2)$ & $78(1)$ \\
$\mathrm{C}(2)$ & $1674(10)$ & $1689(7)$ & $7996(2)$ & $106(2)$ \\
$\mathrm{C}(3)$ & $1324(9)$ & $2369(8)$ & $7364(2)$ & $108(2)$ \\
$\mathrm{C}(4)$ & $2999(7)$ & $4400(7)$ & $7900(2)$ & $76(1)$ \\
$\mathrm{C}(5)$ & $2696(7)$ & $3177(6)$ & $9008(2)$ & $79(1)$ \\
$\mathrm{C}(6)$ & $837(7)$ & $4270(5)$ & $9123(2)$ & $70(1)$ \\
$\mathrm{C}(7)$ & $726(6)$ & $4977(5)$ & $9764(2)$ & $58(1)$ \\
$\mathrm{C}(8)$ & $-1010(8)$ & $5804(5)$ & $9969(2)$ & $73(1)$ \\
$\mathrm{C}(9)$ & $-1194(8)$ & $6342(5)$ & $10584(2)$ & $82(1)$ \\
$\mathrm{C}(10)$ & $401(7)$ & $6069(6)$ & $10994(2)$ & $81(1)$ \\
$\mathrm{C}(11)$ & $2126(7)$ & $5309(5)$ & $10810(2)$ & $77(1)$ \\
$\mathrm{C}(12)$ & $2342(7)$ & $4761(5)$ & $10186(2)$ & $66(1)$ \\
$\mathrm{C}(13)$ & $4478(8)$ & $3879(6)$ & $9358(2)$ & $86(1)$ \\
& & & & \\
\hline & & & &
\end{tabular}


Table 3. Bond lengths $[\AA]$ and angles $\left[{ }^{\circ}\right]$ for rovis7.

\begin{tabular}{|c|c|c|c|}
\hline $\mathrm{O}(1)-\mathrm{C}(4)$ & $1.323(5)$ & & \\
\hline $\mathrm{O}(1)-\mathrm{C}(3)$ & $1.449(6)$ & & \\
\hline $\mathrm{O}(2)-\mathrm{C}(4)$ & $1.181(5)$ & & \\
\hline $\mathrm{O}(3)-\mathrm{C}(12)$ & $1.351(5)$ & & \\
\hline $\mathrm{O}(3)-\mathrm{C}(13)$ & $1.439(5)$ & & \\
\hline $\mathrm{O}(4)-\mathrm{C}(6)$ & $1.203(4)$ & & \\
\hline $\mathrm{C}(1)-\mathrm{C}(2)$ & $1.503(7)$ & & \\
\hline$C(1)-C(4)$ & $1.520(6)$ & & \\
\hline$C(1)-C(5)$ & $1.528(6)$ & & \\
\hline$C(2)-C(3)$ & $1.467(6)$ & & \\
\hline$C(5)-C(13)$ & $1.487(6)$ & & \\
\hline$C(5)-C(6)$ & $1.519(6)$ & & \\
\hline$C(6)-C(7)$ & $1.476(5)$ & & \\
\hline$C(7)-C(8)$ & $1.381(6)$ & & \\
\hline$C(7)-C(12)$ & $1.387(5)$ & & \\
\hline $\mathrm{C}(8)-\mathrm{C}(9)$ & $1.379(6)$ & & \\
\hline$C(9)-C(10)$ & $1.369(6)$ & & \\
\hline $\mathrm{C}(10)-\mathrm{C}(11)$ & $1.336(6)$ & & \\
\hline $\mathrm{C}(11)-\mathrm{C}(12)$ & $1.403(5)$ & & \\
\hline $\mathrm{C}(4)-\mathrm{O}(1)-\mathrm{C}(3)$ & $110.9(4)$ & $\mathrm{O}(4)-\mathrm{C}(6)-\mathrm{C}(5)$ & $123.1(4)$ \\
\hline $\mathrm{C}(12)-\mathrm{O}(3)-\mathrm{C}(13)$ & $114.5(3)$ & $C(7)-C(6)-C(5)$ & $114.7(4)$ \\
\hline$C(2)-C(1)-C(4)$ & $104.2(4)$ & $\mathrm{C}(8)-\mathrm{C}(7)-\mathrm{C}(12)$ & $118.2(4)$ \\
\hline$C(2)-C(1)-C(5)$ & $114.6(4)$ & $C(8)-C(7)-C(6)$ & $121.4(4)$ \\
\hline $\mathrm{C}(4)-\mathrm{C}(1)-\mathrm{C}(5)$ & $114.1(4)$ & $C(12)-C(7)-C(6)$ & $120.4(4)$ \\
\hline $\mathrm{C}(3)-\mathrm{C}(2)-\mathrm{C}(1)$ & $104.7(4)$ & $\mathrm{C}(7)-\mathrm{C}(8)-\mathrm{C}(9)$ & $121.6(4)$ \\
\hline $\mathrm{O}(1)-\mathrm{C}(3)-\mathrm{C}(2)$ & $107.9(4)$ & $\mathrm{C}(10)-\mathrm{C}(9)-\mathrm{C}(8)$ & $118.9(4)$ \\
\hline $\mathrm{O}(2)-\mathrm{C}(4)-\mathrm{O}(1)$ & $121.9(5)$ & $\mathrm{C}(11)-\mathrm{C}(10)-\mathrm{C}(9)$ & $121.4(4)$ \\
\hline $\mathrm{O}(2)-\mathrm{C}(4)-\mathrm{C}(1)$ & $128.5(5)$ & $\mathrm{C}(10)-\mathrm{C}(11)-\mathrm{C}(12)$ & $120.4(4)$ \\
\hline $\mathrm{O}(1)-\mathrm{C}(4)-\mathrm{C}(1)$ & $109.5(4)$ & $\mathrm{O}(3)-\mathrm{C}(12)-\mathrm{C}(7)$ & $122.8(4)$ \\
\hline$C(13)-C(5)-C(6)$ & $107.9(4)$ & $\mathrm{O}(3)-\mathrm{C}(12)-\mathrm{C}(11)$ & $117.7(4)$ \\
\hline$C(13)-C(5)-C(1)$ & $113.9(4)$ & $C(7)-C(12)-C(11)$ & $119.5(4)$ \\
\hline$C(6)-C(5)-C(1)$ & $113.3(4)$ & $\mathrm{O}(3)-\mathrm{C}(13)-\mathrm{C}(5)$ & $113.3(4)$ \\
\hline $\mathrm{O}(4)-\mathrm{C}(6)-\mathrm{C}(7)$ & $122.2(4)$ & & \\
\hline
\end{tabular}

